\title{
Resonance Methods in Quadrupole Ion Traps
}

Dalton T. Snyder'; Wen-Ping Peng ${ }^{1,2} ;$ R. Graham Cooks ${ }^{1 *}$

Keywords: quadrupole ion trap; resonances; quadrupole potential; secular frequency; nonlinear field 


\section{Abstract}

The quadrupole ion trap is widely used in the chemical physics community for making measurements on dynamical systems, both intramolecular (e.g. ion fragmentation reactions) and intermolecular (e.g. ion/molecule reactions). In this review, we discuss linear and nonlinear resonances in quadrupole ion traps, an understanding of which is critical for operation of these devices and interpretation of the data which they provide. The effect of quadrupole field nonlinearity is addressed, with important implications for promoting fragmentation and achieving unique methods of mass scanning. Methods that depend on ion resonances (i.e. matching an external perturbation with an ion's induced frequency of motion) are discussed, including ion isolation, ion activation, and ion ejection.

\section{Introduction}

Quadrupole ion traps are widely used devices for chemical analysis. Typically traps are used to study external dynamical systems like monitoring chemical reactions in a reactor [1] or the composition of the atmosphere [2, 3]. In contrast, 
before ion traps were used for mass analysis, physicists used them as storage devices for studies of trapped ions $[4,5]$. Similarly, the chemical physics community has used traps largely for the examination of internal dynamic systems, that is, reactions or other chemical or physical phenomena that take place within the mass spectrometer. For example, ion traps have been used for studying photodissociation dynamics [6, 7], ion/molecule reactions [8], ion cluster formation [9-12], and to follow induced ion motion using ion tomography $[13,14]$. Other increasingly popular applications of ion traps in chemical physics are for quantum information processing [15-22], production and study of Coulomb crystals [23-26], and ultraviolet/visible and infrared spectroscopy of cold ions [27-32].

The topic of this review is resonances in quadrupole ion traps, which are key aspects of both routine and advanced operation of these devices as mass spectrometers. They significantly contribute to the behavior of many of the massselective operations that can be performed, including ion isolation, ion activation and ion ejection, while simultaneously encoding mass-to-charge ratio information.

Resonances are a common phenomenon in chemical physics. They determine transitions between allowed quantum spin states [15, 33], electronic transitions for molecules irradiated by electromagnetic waves [34], nuclear energy transitions (the Mossbauer effect) [35], nuclear magnetic resonance transitions [36], electron paramagnetic resonance transitions $[37,38]$, and certainly much more.

In a simple macroscopic example, a child on a swing, having a particular frequency of motion, can be resonantly excited by exerting a force in a timedependent manner that corresponds to the pseudo-pendulum's frequency of motion 
[39], consequently resulting in a greater amplitude of motion. This type of resonant phenomenon is known as a parametric resonance because it exhibits an energy transfer which causes an instability phenomenon, and the effect is utilized in many fields, e.g. optics [40] and in quadrupole ion traps [41].

Resonance frequencies of ions in quadrupole ion traps can be determined analytically via Mathieu equations, along with the resonance effects using the specific case of the Hill equation when the force on the system takes a sinusoidal time profile. Consequently, the Mathieu equations can be used to predict ion motion and correlate physically-meaningful quantities (e.g. mass) with, for example, instability in ion motion. Unlike the pendulum, an ion's frequency of motion in a quadrupole ion trap is mass-to-charge-dependent. This fortunate relationship between frequency and mass-to-charge allows resonances to be used for massselectively interrogating ion populations for various purposes i.e. for performing ion isolation, ion activation, and ion ejection.

\section{Quadrupole Ion Trap: Quadrupolar and Higher-order}

\section{Resonances}

\subsection{The quadrupole field \& Mathieu parameters}

A pure "quadrupole field" is produced when four infinitely long electrodes with the appropriate hyperbolic cross sections are arranged at the corners of a square, as in Figure 1a. The quadrupolar (quadratic) nature of the potential dictates 
that the force on a point charge in the electric field varies linearly with the distance from the quadrupole center. Because of this relationship, the field is not quadrupolar but rather the potential is. Nonetheless, it is common to refer to "quadrupolar fields," despite the misnomer.

Equations governing ion motion in a quadrupole field are described in Quadrupole Mass Spectrometry and Its Applications [42] as well as Quadrupole Ion Trap Mass Spectrometry [41] and are taken from Knight [43]. Here we will restrict our discussion to the most important equations in this derivation. In a quadrupole device the potential varies quadratically according to $\phi_{x, y}=\frac{\phi_{0}}{2 r_{0}^{2}}\left(x^{2}-y^{2}\right) \quad$ Eq. 1 where $\mathrm{x}$ and $\mathrm{y}$ are the dimensions of the quadrupole field, $\phi$ is the potential at a given point within the field, and $\mathrm{r}_{0}$ is the half distance between opposite rods. Because the potential varies quadratically from the center of the quadrupole, the derivative of the potential, the electric field strength, varies linearly with respect to distance. The Mathieu differential equation [44] can be used to describe the electric field in the ion trap and its solution can be used to determine the ion motion in the electric field as follows, $\frac{d^{2} u}{d \xi^{2}}+\left(a_{u}-2 q_{u} \cos 2 \xi\right) u=0 \quad$ Eq. 2 where $\mathrm{u}$ is a displacement, $\xi$ is a parameter related to time $\mathrm{t}$ by $\xi=\Omega \mathrm{t} / 2, \Omega$ is the angular frequency of the main trapping radiofrequency (rf) signal, and $a_{u}$ and $q_{u}$ are dimensionless Mathieu parameters. The values of the Mathieu parameters determine the characteristics (especially the stability) of the trajectories of ions 
within the quadrupole field. For the case where the spacing in $\mathrm{x}$ and $\mathrm{y}$ are identical (i.e. $x_{0}=y_{0}=r_{0}$ ) in a $2 D$ linear ion trap, the Mathieu parameters are defined by:

$a_{x}=-a_{y}=\frac{8 z U}{m r_{0}^{2} \Omega^{2}} \quad$ Eq. 3

$q_{x}=-q_{y}=\frac{-4 z V}{m r_{0}^{2} \Omega^{2}} \quad$ Eq. 4

where $z$ is the elementary charge, $\mathrm{U}$ is the DC potential applied to the rods in a quadrupolar manner (as in Figure 1a where the trapping potential is applied in a quadrupolar manner), and $\mathrm{V}$ is the zero-to-peak amplitude of the trapping $\mathrm{rf}$ potential, and $\mathrm{m}$ is the mass of the ion.

The Mathieu parameters $a_{u}$ and $q_{u}$, where $u$ is a quadrupole dimension $(x, y$, $\mathrm{r}$, or z), determine regions of ion stability illustrated by a Mathieu stability diagram as shown in Figure 1b. Any ions with $m / z$-dependent Mathieu parameters that fall within the enclosed region will have stable trajectories and thus may be trapped successfully; other ions will be ejected or will crash into the electrodes. Usually ion traps are operated without trapping DC potentials on the quadrupole rods, so $a_{u}=0$, putting all ions on the $\mathrm{q}_{\mathrm{u}}$ axis. Because both $\mathrm{a}_{\mathrm{u}}$ and $\mathrm{q}_{\mathrm{u}}$ are inversely proportional to $m / z$, ions will be distributed on the $q$ axis as a reciprocal of their $m / z$ values, with higher $m / z$ ions falling at lower $\mathrm{q}_{\mathrm{u}}$ and lower $m / z$ ions residing at higher $\mathrm{q}_{\mathrm{u}}$. This distribution results in higher mass dispersion at low q but lower mass dispersion at high q, which is useful for mass analysis and other ion manipulations.

Each ion also has an $m / z$-dependent pseudopotential well depth, calculated at low $\mathrm{q}_{\mathrm{u}}$ using the Dehmelt approximation [45],

$D_{x, y}=\frac{q_{u} V}{4} \quad$ Eq. 5 
where $D_{x, y}$ is the pseudopotential well depth (note that the constant in the denominator will depend on device geometry). This approximation is only valid for $\mathrm{q}_{\mathrm{u}}<0.4$, although it remains a reasonable approximation up to $\mathrm{q}_{\mathrm{u}}=0.7$ [46]. Exact pseudopotential well depth can be calculated numerically for different geometries, as shown in Figure 1c $[47,48]$ for a specialized square sinusoidal ion trap (SIT) configuration. The well depth describes a harmonic oscillator trapped in a potential well. If the ion obtains kinetic energy equaling or exceeding the well depth, the ion can escape from the trap.

\subsection{Quadrupole ion trap geometries}

There are two standard geometries for producing a quadrupole electric field for trapping and mass analyzing ions. The first, developed by Wolfgang Paul in the 1950s [4], is the 3D quadrupole ion trap (QIT), also known as the quadrupole ion storage device (QUISTOR). As shown in Figure 2, the QIT is constructed from a ring electrode and two endcap electrodes. Typically, the trapping rf potential is applied to the ring and the endcaps are used for ion injection, ejection, and frequency-based ion manipulations using low-voltage ac waveforms. Schwartz [49], Hager [50], and Bier [51] developed 2D linear ion traps (LIT), which have four parallel rods with hyperbolic cross sections for radial trapping and endcaps with DC potentials or multiple-section quadrupole rods with DC offsets for axial trapping. The LIT has two principal performance advantages over the QIT: 1) increased ion storage capacity (hence a reduction in space charge effects) and 2) dramatically increased ion injection efficiency. 
Many geometrically simplified versions of these devices exist (Figure 2), usually developed for ease of fabrication and miniaturization or purposeful introduction of higher-order fields [52]. For example, the cylindrical ion trap, simplified from the 3D ion trap, has electrode surfaces that are planar rather than hyperbolic [53-57]. Similarly, the rectilinear ion trap also has planar surfaces, but is a variant of the 2D linear ion trap [58]. The toroidal ion trap is produced by rotating a 3D trap about an axis parallel to its trapping region [59-62]. The halo trap is a simplified variant of the toroidal trap with planar electrode structures [63]. While these simplified traps benefit from easier fabrication methods, they inherently introduce higher-order fields that can degrade (and occasionally enhance) performance, as discussed later.

\subsection{Ion dynamics and nonlinear field contributions}

Trapped ions will behave according to their Mathieu parameters $a_{u}$ and $q_{u}$, but these parameters not only determine their trajectory stability, they also determine the actual ion motion within the trap. Ion motion is described principally by a fundamental secular frequency

$\omega_{u, 0}=\frac{\beta_{u} \Omega}{2} \quad$ Eq. 6

where the parameter $\beta_{\mathrm{u}}\left(0 \leq \beta_{\mathrm{u}} \leq 1\right)$ is calculated using a continued-fraction expression in terms of $\mathrm{a}_{\mathrm{u}}$ and $\mathrm{q}_{\mathrm{u}}$

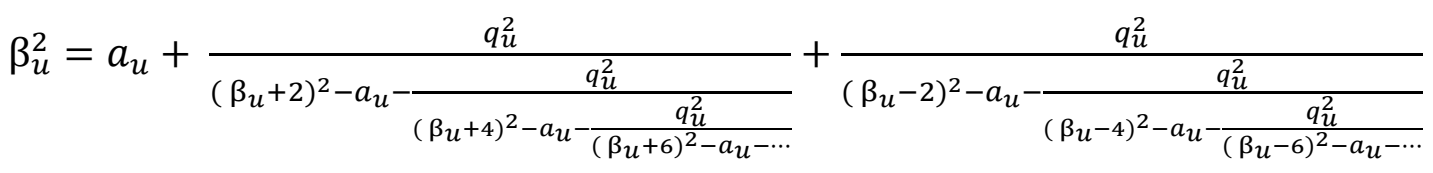

Eq. 7 
By using the Dehmelt approximation, we can simplify equation 7 to

$\beta_{u}=\left(a_{u}+\frac{q_{u}^{2}}{2}\right)^{1 / 2} \quad$ Eq. 8

so that we can approximate the secular frequency from the experimental

parameters giving (with $a_{u}=0$ )

$\omega_{u, 0}=\frac{\sqrt{2} z V}{\Omega u_{0}^{2} m} \quad$ Eq. 9

Thus we see that an ion's secular frequency is approximately inversely proportional to its $m / z$ value. In this regard, an ion trapped in a quadratic potential is more akin to a mass at the end of a spring than to a mass on a pendulum. In the case of the spring, the frequency of oscillation is inversely proportional to the square root of the mass, whereas the pendulum's frequency has no mass dependence.

Ions also have parametric resonances,

$\omega_{\mathrm{u}, \mathrm{n}}=\left|\mathrm{n}+\beta_{\mathrm{u}}\right| \Omega / \mathrm{K} \quad-\infty<\mathrm{n}<\infty ; \mathrm{K}=1,2, \ldots$ Eq 10

where $\mathrm{K}$ is the order of the resonance $[64,65]$. The first order parametric resonance is twice the fundamental secular frequency.

More generally an ion will have multiple frequency components of motion in a pure quadrupole field, described by

$\omega_{u, n}=\left|2 n+\beta_{u}\right| \Omega / 2 \quad-\infty<n<\infty \quad$ Eq 11

where $\mathrm{n}$ is an integer, and where the amplitudes of each component varies.

Figure 3a shows a calculated frequency spectrum of an ion oscillating in a pure quadrupole field [66] at a q value of $0.68\left(\omega_{\mathrm{z}}=.27 \mathrm{MHz}, \mathrm{a}_{\mathrm{z}}=0\right)$. The primary frequency at which the ion oscillates in the trap is the secular frequency $\omega_{\mathrm{u}, 0}$ but there are smaller contributions from sideband frequencies $\Omega-\omega, \Omega+\omega$, and $2 \Omega-\omega$. 
The sideband frequencies result from interference of the ion's secular frequency and the driving rf frequency. However, note that no driving frequency, $\Omega$, is observed in this Fourier analysis. As the ion approaches the right-hand side boundary of the stability diagram $\left(q_{u}=0.908, \beta_{u}=1\right)$, the sideband oscillations become much more prominent and dominate ion motion over the secular frequency [67].

In practice, pure quadrupole fields do not exist because of real-world constraints. For example, electrode misalignments exist which can degrade the electric field [68]. Electrodes cannot be manufactured to have perfectly smooth surfaces [69] or ideal hyperbolic cross sections, nor can they be infinitely long. Furthermore, slits and apertures must be integrated into the hyperbolic electrodes for ion introduction, ion injection, and often for ionization [70, 71]. Because of these constraints, higher-order fields are introduced.

A higher-order field is described by both its order as well as its multipole coefficient [72-74]. For example, 0, 1, 2, 3, 4,... stand for monopole, dipole, quadrupole, hexapole, octopole,..., components, respectively. Importantly, the potential in the field varies as $\mathrm{r}^{\mathrm{n}}$, where $\mathrm{n}$ is the order of the field. Mathematically, multipole coefficients originate from the spherical distribution of the potential in the 3D trap

$$
\Phi(\rho, 0)=\Phi_{0} \sum_{n=0}^{\infty} A_{n} \frac{\rho^{n}}{r_{0}^{n}} P_{n}(\cos \theta) \quad \text { Eq. } 12
$$

Here $\rho$ and $\theta$ are spherical coordinates, $A_{n}$ is the multipole coefficient of order $\mathrm{n}$ and $P_{n}(\cos \theta)$ is a Legendre polynomial. In a pure quadrupole field $A_{2}=1$ and $A_{n}=0$ for all other $\mathrm{n}$, implying that the electric field strength increases linearly and the potential increases quadratically with the distance from the quadrupole center. 
However, when higher-order fields are added, the potential deviates from ideality. For example, the octopole $\left(\mathrm{A}_{4}\right)$ potential exhibits an $\mathrm{r}^{4}$ dependence so that the electric field exhibits an $r^{3}$ dependence.

Here we are concerned with the effect of higher-order fields on ion frequencies of motion, which can be determined either numerically or measured experimentally by laser or DC pulse tomography $[13,75,76]$. Both the magnitude and direction of the effect are determined by the nature of the higher-order field, that is, by whether the higher-order field is mathematically odd or even. Odd-order fields (e.g. hexapole, $\mathrm{n}=3$ ) cause the potential in the trap to deviate in a mathematically odd manner, where the potential increases from ideality near one electrode but decreases near the opposite electrode. The net result is that ions occupy regions of weaker field strength than they would had the field been purely quadrupolar [66], thereby lowering their secular frequencies. For even-order fields (e.g. octopole, $n=4$ ), the opposite is true. The electric field strength will be higher than a pure quadrupole field, particularly near the electrodes where higher-order fields are more prominent, thereby raising the ions' secular frequencies [77-79]. In summary, odd-order fields decrease secular frequencies, though only slightly, and even-order fields increase or decrease secular frequencies (compared to a pure quadrupole), depending on the nonlinear field polarity. The resulting effect on resonance methods will be discussed later.

Higher-order fields additionally cause the introduction of further frequency components to ion motion [66]. As shown in Figure 3b, the addition of (for example $20 \%$ ) hexapole field introduces even and odd overtone frequencies (e.g. $2 \omega_{\mathrm{u}, 0}, 3 \omega_{\mathrm{u}, 0}$, 
etc.), with the driving $\mathrm{rf}$ frequency $\Omega$, and even and odd sideband frequencies (e.g. $\Omega$

$+2 \omega_{\mathrm{u}, 0}, \Omega+3 \omega_{\mathrm{u}, 0}$ ). Introduction of (for example $20 \%$ ) octopole field induces only odd overtones and their sidebands (Figure 3c) [80].

Although these additional frequencies can complicate mass analysis (after all, ions are mass-selectively interrogated using their secular frequencies), the more significant consequence of higher-order fields is ion frequency shifts. But what effect does this have on mass-selective operations?

\subsubsection{Effect of higher-order fields on mass analysis}

The most basic and significant mass-selective operation is mass analysis, that is, scanning ions out of the trap in order of $m / z$ to determine the ions' $m / z$ values. Typically, this is done using resonance ejection, in which a small supplementary ac field is applied in a dipolar manner to the endcap electrodes (3D trap) or $\mathrm{x}$ electrodes (2D trap) [81, 82]. When the ac frequency matches the frequency of motion of ions of a given $m / z$ value, then those ions will gain kinetic energy and increase their radial and/or axial amplitudes in the trap until they are ejected. If the rf amplitude is ramped linearly with time [83], then ions are scanned linearly into the resonance frequency and ejected linearly in order of $m / z$. This process maintains linearity for a pure quadrupole (linear) field. However, if a non-linear odd-order field such as a hexapole field is introduced, then the ion's secular frequency will decrease as the ions distance from the quadrupole center [79] changes. This variation of frequency as a function of distance from the center is typically much smaller than it is for even-order field distortion [77, 84], however 
the result is that ion secular frequencies will either decrease or increase with the radial distance from the trap center depending on the type of field distortion (Figure 4a). Note that traps with both axial and radial symmetry generally only contain even-order fields in which ion secular frequencies will increase with the radial distance for a positive field contribution $[85,86]$. The corresponding effect on mass spectral performance with resonance ejection is that, for traps with positive even-order fields, resolution is increased when scanning the rf amplitude from low to high, but decreased when scanning from high to low, as shown in Figure 4b [8789]. This is because as ions are brought to the resonance ejection working point, they increase their amplitudes in the trap. When scanning the rf amplitude forward, the ion secular frequencies, which increase with the rf amplitude, shift towards the supplementary ac frequency as the ions are being ejected because of the even-order fields, thereby increasing their rate of ejection. On the other hand, when the rf amplitude is scanned downward, the ion secular frequencies shift away from the supplementary ac frequency as they are being ejected, thereby delaying the process. Despite the improvement in resolution, these frequency shifts can also result in a loss in calibration linearity (i.e. $m / z$ vs. time). Not only do the shapes of the electrodes alter the nonlinear field contributions, but the inter-electrode distance and alignment also alter the field, which can then have a negative effect on resolution (Figure 4c) [90].

Resolution is not the only mass spectral characteristic altered by nonlinear field contributions. Chemical mass shifts can also be induced (Figure 4d) [91, 92]. As an ion's secular frequency changes with its distance from the trap center, it's 
apparent $m / z$ will also change. Each $m / z$ correlates with a particular frequency of motion, so any frequency shift induced by higher order fields will have a corresponding effect on peak location in the mass spectrum, resulting in mass shifts. Space charge, in which Coulombic repulsion affects ion motion, can have a similar effect on ion frequencies [93-96], which can degrade both resolution and mass accuracy. A third, similar parameter that affects resolution and mass accuracy is the momentum of the ions, which is modulated in ion traps with a light bath gas (e.g. helium) [97]. For example, additional damping from collisions with gas molecules delays the onset of ion ejection (the voltage needed for resonant ejection), and this relationship is highly dependent on the ion's qu parameter.

\subsubsection{Effect of higher-order fields on ion excitation}

The ion excitation process is also affected by higher-order field components. There are many ways to kinetically or internally excite ions in an ion trap [82,98102]. The most typical method though, is resonance excitation [82]. In this method, ions are simply excited at their secular frequencies by applying a low-amplitude (a few hundred millivolts) dipolar ac signal matching the ion's secular frequency. The resonated ions will then gain kinetic energy and collide with bath gas molecules, gaining internal energy until dissociation channels are accessed which generate fragments reminiscent of the precursor ion. However, in the presence of higherorder fields the ion's secular frequency will shift as a function of the radial amplitude in the trap [103], thereby causing the ion to go out of resonance with the excitation. This frequency shift can be beneficial, as it allows higher ac amplitudes to 
be used for excitation which may increase fragmentation efficiency. On the other hand, there are methods to compensate for any ion frequency shift, some of which involve exciting the ion with a frequency sweep ('chirp'), a band of frequencies [104], or a high-amplitude off-resonance frequency [105].

\subsection{Nonlinear Resonances}

Not only do higher-order fields affect ion secular frequencies, but they also induce nonlinear resonances $[66,106,107]$. At these points ions pick up energy and their amplitudes of motion increase hyperbolically; that is, their trajectories can become quite unstable. The general resonance condition is

$n_{r} \omega_{r}+n_{z} \omega_{z}=v \Omega \quad$ Eq. 13

where $n_{r}, n_{z}$, and $v$ are integers. Symmetry forces $n_{r}$ and $n_{z}$ to be even for symmetric traps and even order fields; however, $\mathrm{n}_{\mathrm{z}}$ may be either even or odd for odd-order (nonsymmetrical) fields.

As an example, consider the case where $n_{r}+n_{z}=3$, i.e. the hexapolar resonance. In this case a nonlinear resonance line is observed at, for example, $\beta_{z}=$ $2 / 3$, depending on the values of $n_{r}$ and $n_{z}$. For a second example where $n_{r}+n_{z}=4$, octopolar resonances are observed. When $\mathrm{n}_{\mathrm{r}}=0$ and $\mathrm{n}_{\mathrm{z}}=4$, a resonance is observed at $\beta_{\mathrm{z}}=1 / 2$. Many other resonance lines are observed (for example, a weak dodecapole resonance at $\beta_{\mathrm{z}}=1 / 3$ ), but we limit our discussion here to these octopole and hexapole resonances lines, which are the strongest and have the most utility.

Nonlinear resonance lines are important to understand because they can significantly alter mass spectra. For example, artifact peaks, often called 'ghost' 
peaks, can be observed during a scan of the rf amplitude when obtaining a full scan mass spectrum. While most ions will be ejected when their Mathieu $\mathrm{q}_{\mathrm{u}}$ values match the $q_{u}$ value of the resonance ejection signal or when they reach the stability boundary $\left(q_{u}=0.908\right)$, other ions can be ejected when they pass through nonlinear resonance lines, particularly when the ion cloud is large (e.g. when sample concentration is high or when ions have previously been resonantly excited). Typically, 'ghost' peaks are broader than other mass spectral peaks and so can often be distinguished from boundary and especially from resonance ejection peaks. Other methods of distinguishing all of these potential causes of mass spectral peaks have been investigated [108].

So-called 'black holes' or 'canyons' are another consequence of the imposition of higher-order fields on the standard quadrupole field [109-112]. Unexpected black holes appear most prominently when ions are accumulated in the trap or are formed inside the trap and happen to fall on a non-linear resonance. Any activity that places ions close to the electrodes where higher-order fields are more prominent can enhance the observation of these effects. In tandem mass spectrometry spectra (MS/MS), product ions that are produced and fall on a nonlinear resonance line are inefficiently stored, resulting in diminished intensity in the normal mass scan (Figure 4e). Similar results have been reported in chemical ionization (CI) mass spectra, particularly when the chemical ionization reagent is introduced in high proportions, where space charge effects push ions into large radial displacements. Full scan mass spectra can be affected when long ion storage times are used. The intensities of ions stored at the nonlinear resonance points have 
been observed to decay [113], and the absolute signal attenuation has been observed to be dependent upon scan direction. Moreover, the ion losses appear reasonably - to be more prominent when the rf is scanned more slowly. Frequency scanning methods (e.g. secular frequency scanning) have also reportedly observed mass spectral changes from black holes, either observed as blank intensity profiles or broadened mass peaks, depending on scan direction [114] (Figure 4f).

\section{Ion Trap Resonance Methods}

We broadly divide ion trap resonance methods into three distinct categories based on their consequences: ion isolation, ion excitation, and ion ejection. The first, isolation, is usually used to decrease chemical noise in mass spectra, to decrease space charge effects, or to select an ion population for ion excitation. In the second, ion excitation, selected precursor ions undergo sufficiently energetic collisions with background gas molecules, causing the generation of product ions which are indicative of the molecular class and/or the structure of the precursor ion. The final step, and perhaps the most fundamental to mass spectrometry, is to measure the mass-to-charge ratios of the ions in the trap, which is usually accomplished by massselectively ejecting them in order of increasing $\mathrm{m} / \mathrm{z}$ into a detector, which subsequently converts the ionic particles to a current and voltage that can be measured and computerized. Measuring ion mass/charge ratios internally using charge detection is also possible. 


\subsection{Isolation}

The isolation step in ion trap mass spectrometers is often overlooked but grants a great deal of flexibility as an analytical tool. Without ion isolation there would be ambiguity in identifying a compound of interest using a fragmentation step due to the difficulty in assigning relationships between precursor and product ions. Moreover, ion isolation allows extension of the dynamic range of the ion trap for isolated analytes; dynamic range in ion traps is limited relative to other mass analyzers because of a limited trapping volume and because the relative contribution of space charge effects decreases with ion velocities. For monitoring chemical reactions, ion isolation is particularly effective for allowing detection of trace quantities of starting material, product, or reaction intermediates.

Modern ion isolation methods are surprisingly complex, often requiring complicated electronics and software for custom waveform calculations and implementation. Ion secular frequencies are utilized by resonantly ejecting particular ions while retaining other ions by removing their frequencies from a multi-frequency resonance ejection isolation waveform. Important performance and operating parameters to consider include $m / z$ isolation width, isolation efficiency, the time required for isolation, waveform amplitude, bandwidth, and complexity, and the pseudopotential well depth of the ion to be isolated compared to other ions in the trap. We note that while isolation techniques are usually performed after ion injection and cooling, they are equally useful for preventing certain ions from being trapped during the ion injection stage, a time-saving trick but with poorer performance compared to in-trap isolation if the trap is not perfectly symmetrical. 
Isolation techniques can be subdivided into methods that 1) modulate the trapping $\mathrm{rf}$ waveform (many of which are not strictly resonance methods), 2) use low-voltage resonance ac waveforms with constant rf parameters, or 3) a combination of the two.

The first category encompasses rf-only isolation (a nonresonance method, yet still significant enough to include here) in which ions of a particular $m / z$ are isolated by ejecting the ions lower in $m / z$ at the upper stability boundary $(q=0.908)$ and also those of higher $m / z$ near the lower stability boundary $(\mathrm{q} \sim 0$ ) where their kinetic energies outweigh their well depths. This is accomplished by increasing and then decreasing the rf amplitude $[83,115]$. The quadrupole ion trap can further be used as a quadrupole ion store (QUISTOR) by operating it in rf/dc mode [116], wherein the rf and dc potentials are fixed so that the ion to be isolated is placed at the apex of the Mathieu stability diagram (approximately $\beta_{\mathrm{z}}=1, \beta_{\mathrm{r}}=0$ in Figure 1b), causing the trajectories of all other species to become unstable. Similarly, the $\mathrm{rf} /$ resonance ejection method uses the already-discussed rf ramp for ejection of low-mass ions but adds a supplementary ac signal at a constant Mathieu $\mathrm{q}_{\mathrm{u}}$ value just below that of the ion to be isolated, thereby removing the higher $m / z$ ions during the rf ramp [117]. An alternative approach only uses the resonance signal, not the stability boundary, to eject unwanted ions [118]. In this case the rf signal is ramped so that the lower $m / z$ ions are ejected up to the resonance point, at which time the supplemental ac is switched off and the rf is increased to bring the ion to be isolated above the q value of the resonance point, and finally the resonance point is restored and the rf amplitude again ramped (this time from high to low) to eject 
higher $m / z$ ions. By tuning the frequencies and amplitudes of the resonance signal and also by using fast (low resolution) and slow (high resolution) ejection scans, high resolution isolation can be accomplished [119].

Besides using the rf amplitude for ion isolation, the $m / z$ dependence of each ion's secular frequency in an ion trap can be exploited. Applying a dipolar ac field in resonance with a particular ion's secular frequency will, at the very least, excite that ion and if the amplitude and time of application are high enough, the ion can be mass-selectively ejected. The simplest way to effect frequency-based isolation is to perform a frequency sweep, or 'chirp' [115, 120-122], whereby the supplemental ac frequency, either chosen to be mono- or polychromatic, is ramped through ion secular frequencies. Unit isolation width has not been demonstrated using a frequency chirp, and broadband waveforms have largely replaced the chirp as the primary method of broadband excitation.

Broadband waveforms can be synthesized by the stored waveform inverse Fourier transform, a sum of sine waves approach, filtered noise filtering, and frequency modulation and mixing. The stored waveform inverse Fourier transform (SWIFT), adopted from the Fourier transform ion cyclotron instrument [123-126], is perhaps the most difficult method because it requires specialized circuitry and complex computations to implement. In brief, a band of secular frequencies (Figure 5a) are inverse Fourier transformed from the frequency domain to the time domain (Figure 5b) in order to obtain a complex waveform that can near-simultaneously excite a wide swath of ions, hence making this method more time-efficient than a frequency sweep. If a 'notch' is incorporated in the frequency domain, those 
frequencies are not incorporated into the waveform and so the corresponding ions will be retained in the ion trap [127]. Perhaps the simplest example of ion isolation using a multi-frequency waveform is that which uses only two frequencies [128], that is, dual frequency isolation. In principle one should be able to eject a wide range of $m / z$ values higher than the target ion by using a high-amplitude, low frequency sinusoid, and also eject ions lower in $m / z$ with a high-amplitude, high frequency sinusoid. In this case, it is the amplitude of the waveform that provides the necessary broadband ejection capabilities, but isolation efficiency is understandably low.

Although the SWIFT technique is more time-efficient than frequency sweeping, experimental results have demonstrated that similarities still exist. For example, not all frequencies are present at all times in a SWIFT waveform. This can lead to problems with using ion injection waveforms because unwanted ions will enter the trap at some time during the application of SWIFT. A sum of sines approach with appropriate phase distribution, currently implemented in Thermo Finnigan mass spectrometers, alleviates this issue because all frequency components are present at all times [129]. Unit isolation width can be accomplished using this method.

Other simpler, but still high-performance, methods of performing broadband excitation of ions do exist. For example, Hilger et al. demonstrated a simpler broadband excitation method wherein a broadband waveform produced by frequency modulation was mixed with a sine wave with frequency matching the target ion's secular frequency (Figure 5c). The performance was shown to be 
equivalent to apex and SWIFT isolation, though it required a longer isolation time than the apex method [130]. The filtered noise method is another broadband technique for ion excitation (Figure 5d) [131, 132]. A broadband filtered noise waveform can be produced by a function generator, and frequencies can be individually removed in a waveform editor, allowing for 'notched' waveforms to be used for isolation. Note that, just as in SWIFT isolation and frequency modulation and mixing, multiple 'notches' can be used to simultaneously isolate multiple nonadjacent $m / z$ ions. In practice, filtered noise is problematic because of the randomness of the noise, which can result in holes in the isolation window. As such, current implementations of filtered noise are more akin to a sum of sine waves.

Another way to effectively isolate and trap ions with a predetermined massto-charge ratio is to apply the auxiliary voltage to the end cap electrodes, where the auxiliary voltage has a frequency spectrum with a first notch at the secular frequency of the object ions and a second notch at a beat frequency to expel nonobject ions still remaining in the ion trap space while the RF voltage is applied to the ring electrode to trap the object ions [133].

Perhaps the best method for ion isolation, achieving unit isolation width, was demonstrated by Wells et al., who combined the broadband techniques with rf ramp methods [134]. A broadband waveform was first used for coarse ejection of ions over a broad mass range during the ionization stage. Subsequently the ions with $m / z$ values below the target ion were ejected by moving the ion to the stability boundary while simultaneously applying a resonance ejection signal with frequency approximately half the driving $\mathrm{rf}$ frequency. Ions with $\mathrm{m} / \mathrm{z}$ higher than the target ion 
were then ejected by moving the target ion to $\mathrm{q}=0.84$ and applying a broadband waveform covering the higher $m / z$ ions.

In contrast to sinusoidal traps, the digital ion trap (DIT) uses duty cycle manipulation to effect ion isolation (Figure 5e,f) $[135,136]$. The digital trap uses square waves generated by high-frequency switching technology to trap ions. The switching for the DIT can be completely controlled by a computer so that mass scanning is comparably quite simple, while complicated LC tank circuits are often required to produce the high trapping rf voltage for conventional sinusoidal waveform-driven ion traps. For ion isolation in the DIT, the duty cycle of the trapping digital waveform is manipulated so that it is not equal to $50 \%$, which introduces a pseudo-dc potential because the average potential of the trapping waveform will then be nonzero (unlike a sine wave centered at zero). Precisely speaking, the nonzero pseudo-dc component does not change the Mathieu $\mathrm{a}_{u}$ parameters of all the ions in the trap but instead changes the boundary conditions so that a small range of $m / z$ values is trapped (Figure 5f) [137].There are many other advantageous features of digital ion traps associated with the precision and speed with which square wave signals can be controlled, but they cannot be discussed here $[138,139]$.

\subsection{Activation}

The second general use of resonances that we discuss here is for ion activation, the process by which ions' kinetic and/or internal energies are increased, usually resulting in fragmentation. The usual use for activating ions is for generating product (fragment) ions, which can be used for structural characterization or 
unambiguous confirmation of the identity of the precursor ion. However, activation can also be used for other purposes. For example, reactions in the trap can be inhibited by activating ions to reduce the overlap between reactant ions, e.g. in a method known as parallel ion parking [140]. Note that this method can also be used to reduce overfragmentation in photodissociation techniques such as infrared multiphoton dissociation (IRMPD) and ultraviolet photodissociation (UVPD). Reactions and molecular fragmentation can also be induced by colliding reactant ions with surrounding bath gas molecules (e.g. unimolecular dissociation).

Ion activation in quadrupole ion traps is usually effected by a variant of collision-induced dissociation (CID), in which ions are resonantly excited and absorb power from the driving rf, gain kinetic energy, collide with intentionallyintroduced bath gas molecules, and fragment, generating product ions that can then be further fragmented or mass analyzed [141]. The most common method is resonance excitation (Figure 6a), in which a small supplementary dipolar ac waveform at a particular ion secular frequency is applied across the endcaps ( $\mathrm{z}$ direction, 3D trap) or across $\mathrm{x} / \mathrm{y}$ electrode pair (2D linear trap) in order to mass selectively excite a sub-population of ions [81]. Important parameters are the ion's Mathieu qu parameter, which controls CID efficiency through the Dehmelt potential well depth, product ion mass range, product ion collection efficiency, and excitation frequency, and parameters not dependent upon $\mathrm{q}_{\mathrm{u}}$, including excitation time, bath gas pressure, and excitation ac amplitude. A high Mathieu qu parameter for a precursor ion will allow more energy input than low $\mathrm{q}_{\mathrm{u}}$ CID due to higher potential well depths [45] and higher ion oscillation frequencies (higher velocities) but will 
sacrifice product ion mass range $\left(m / z_{\text {product }}>m /\right.$ zprecursor $^{*}$ q $_{\text {precursor }} / 0.908$, where 0.908 corresponds to q value at the right-hand side boundary of the Mathieu stability diagram). CID at low Mathieu qu values increases product ion mass range but limits energy deposition because of lower frequencies of ion oscillation (lower velocities) and because the precursor ion can more easily be ejected. As a result, optimum values of Mathieu $\mathrm{q}_{\mathrm{u}}$ parameters for excitation are $\sim 0.2-0.35$, which balances fractional product ion mass range and adequate energy deposition.

There are many other methods for fragmenting ions over a small mass range. As mentioned previously, ions can be resonantly excited by a single frequency ac wave. A variant which increases CID efficiency is dual direction excitation [142] (Figure 6b), in which ions are excited in both $\mathrm{x}$ and $\mathrm{y}$ in a linear ion trap by applying a single dipolar ac to each pair of electrodes. Because ions are excited simultaneously in both directions, the kinetic energy of the ions increases, thereby promoting more and higher-energy collisions.

A key characteristic of CID is the time required to generate the product ion mass spectra. This is particularly important for applications that require methods on the chromatographic time scale (>10 spectra/sec). Another concern with CID in traps is the lower-mass cutoff imposed by the stability boundary associated with the driving rf. The latter problem can be mitigated by using "pulsed Q dissociation" (PQD) $[100,143]$ (Figure 6c). In PQD, the ion to be excited is placed at a high $\mathrm{q}_{\mathrm{u}}$ value (0.6-0.8) and is resonantly excited with a short ( $100 \mu$ s) pulse with a high amplitude. After a short time delay $(\sim 100 \mu s)$, the rf amplitude is quickly decreased so the ion is at a low $q_{u}$ value. The short sequence of events preserves low mass 
product ions while also leading to higher-energy collisions. Similar methods include high amplitude short time excitation (HASTE) [144] and "fast excitation" CID [145] (Figure 6d), both of which use high excitation amplitudes over a short period of time.

Although the intuitive method of ion activation is through use of onresonance methods, off-resonance methods can be equally appealing because an ion's secular frequency (eq. 6) will shift if the strength of trapping field deviates from linearity, that is, if there is any contribution from higher-order fields. Red-shifted off-resonance large-amplitude excitation (RSORLAE) [146], blue-shifted offresonance CID [105], and dynamic CID (DCID) [147-150] all take advantage of the large distribution of ions that are excited when a high-amplitude ac signal is used. Typically, a red-shifted (i.e. lower than the ion's secular frequency) ac signal is used instead of a signal with frequency matching the ion's secular frequency. The utility of the off-resonance signal is that it 1) allows higher energy deposition than onresonance CID does, and 2) any shift in ion secular frequency caused by introduction of higher-order fields is mitigated because the high-amplitude signal excites ions over a wide $m / z$ range. In the case of DCID, a two-frequency excitation waveform is used. The two frequencies are spaced very closely from one another $(\sim 1 \mathrm{kHz}$ difference) so that a beat frequency develops (Figure 6e). This technique also allows for high energy deposition when compared to conventional on-resonance activation and CID.

Although collisions with background gas molecules are most commonly used, ions can also be forced to collide with a surface in the technique of surface-induced 
dissociation (SID) [98]. The energy absorption profile is significantly different than that for CID. In CID, ions slowly convert kinetic energy into internal energy, usually resulting in labile bond fragmentation, often giving less informative product ions [151]. In SID, the large mass of the collision partner results in high-efficiency kinetic to internal energy conversion despite the low translational energies $(\mathrm{eV})$ involved. For analytes like peptides and proteins, SID is particularly useful because of the extensive backbone cleavage observed. Conventionally, SID employs two separate mass analyzers, one to guide ions into a surface while the products are extracted into the second analyzer (e.g. a second quadrupole mass filter). SID can also be performed in a single quadrupole ion trap by resonantly exciting the precursor ions until they collide with the quadrupole electrodes, thereby inducing fragmentation [152]. Unfortunately, trapping of the product ions is inefficient, so SID has not been extensively implemented in QITs.

So far we have discussed single resonance techniques that fragment ions over a small $\mathrm{m} / \mathrm{z}$ range, but there are also broadband CID methods which fragment ions over a wide range of $m / z$ values. For example, dipolar DC collisional activation [153-155], a non-resonance technique, can fragment ions over a wide $m / z$ range by displacing the entire ion cloud from the center of the trap using a dipolar DC potential. Because ion secular frequencies are not interrogated this is not a massselective method.

A secular frequency scan $[114,156]$, in which the rf frequency and amplitude are held constant (i.e. ion Mathieu q values do not change during the scan), can effect broadband fragmentation of ions. This experiment is useful in combination 
with mass-selective detection of specific fragment ions in single analyzer ion traps.

The result is a precursor ion scan in a single mass analyzer $[157,158]$.

Unfortunately, there is a nonlinear relationship between secular frequency and $m / z$, so if the ac frequency is scanned linearly (the easiest method to implement) then the resonance time will vary with $m / z$. Furthermore, each ion is only on resonance for a very short period of time, limiting energy deposition and hence fragmentation.

Although the stored waveform inverse Fourier transform is more commonly associated with ion isolation, it can also be used for fragmentation [123-126, 159, 160]. The results from broadband SWIFT excitation are comparable to ac frequency scanning excitation; however, the former is more time-efficient. In broadband CID techniques, each mass-selected ion is excited for the duration of the excitation waveform, whereas for the secular frequency scan each ion is only on-resonance when its secular frequency matches the ac frequency. As in isolation, notches can be incorporated into the activation waveform in order to prevent activation of some ion species. Equivalently, a filtered noise field (FNF) can produce notched broadband waveforms $[131,132]$. A useful example of broadband excitation using SWIFT and FNF is for parallel ion parking in ion traps, a technique for consolidating protein charge states [140].

While broadband waveforms provide an efficient means of broadband CID, they are limited in ion traps by the fundamental laws governing ion trap CID as mentioned above, related to the balancing of potential well depth, activation frequency, and fractional product ion mass range. This train of thought has recently been extended to broadband CID in a method termed "multigenerational CID" [161] 
(Figure 6f). In this method the excitation Mathieu $q_{u}$ value is fixed at an optimal value (in the range $0.2-0.35$ ) by setting the ac waveform at the corresponding frequency. The fixed excitation frequency only fragments ions over a limited range, so in order to fragment a wide range of $m / z$ values, the $\mathrm{rf}$ amplitude must be scanned from high to low, thereby fragmenting ions successively from high to low $m / z$. Because each ion is fragmented at the same $q_{u}$, chosen to balance precursor well depth, ion secular frequency (velocity), and fractional product ion mass range, higher intensities of product ions are observed and a wider distribution of product ion $m / z$ values is also observed.

The term 'multigenerational' refers to the characteristic wherein multiple generations of fragment ions are produced from a single scan of the rf amplitude [162]. An equivalent, but experimentally limited Wideband Activation method is available on Thermo ion trap instruments [163]. While Wideband Activation, as commercially implemented, is limited to a 20 Th mass range, multigenerational CID is much more versatile because the rf amplitude is scanned over a larger range, thereby extending the excitation mass range. Useful applications of multigenerational CID and Wideband Activation are included in the practical applications of ion trap mass spectrometry, e.g. in discrimination of designer drugs, which have similar $\mathrm{MS}^{2}$ spectra but also often have unique $\mathrm{MS}^{3}$ fragments. Molecules with hydroxyl, carbonyl, and sulfate functional groups, which often give uninformative neutral losses of water, carbon dioxide, and sulfite, respectively, are fragmented more extensively with either of these newer methods. The spectra that are observed with a single sequence of ion injection, CID, and mass scanning contain 
combinations of ions from the fragment ions which would otherwise be observed in discrete $\mathrm{MS}^{2}$, MS ${ }^{3}$, etc. spectra.

The multigenerational effect can also be observed by using a frequency sweep of the resonance ejection signal while keeping the rf parameters constant [121]. The observed fragmentation patterns are nearly identical to the rf ramp case. However, because the precursor and many generations of product ions lie on different locations of the Mathieu q axis, their fractional product ion mass ranges will differ, as will their excitation frequencies.

The digital ion trap can also be used to perform MSn, and the implementation is similar to that using sinusoidally-powered ion traps. Major differences are the naturally deeper potential well in the DIT because of the higher average potential of the trapping waveform as well as the reduced mass range (boundary at $\mathrm{q}=0.712$ compared to $\mathrm{q}=0.908$ for $\mathrm{rf}$ trap) although there are DIT methods of broadening this range. The first implementation of CID in the DIT used conventional sinusoidal dipolar ac waveforms [164], but further investigations yielded a more appropriate digital method of CID wherein the digital resonance excitation signal, obtained by dividing down the digital trapping waveform, was duty cycle modulated to provide a dipolar waveform for CID. For example, the digital trapping waveform can be divided by 3 to obtain a nonlinear resonance condition at $\beta_{z}=2 / 3$ (i.e. a double resonance, explained in the ejection section), which is used for ion ejection. Dipolar DC CID was accomplished by increasing the duty cycle of the supplemental digital waveform, introducing a radial DC component so as to displace the ion cloud from the center of the trap, thereby inducing rf heating and thus fragmentation. The CID 
efficiencies approach $100 \%$ in some cases, but this technique is not mass-selective so that the ions to be activated must first be isolated to preclude spectral complexity. Further work showed that the frequency of the dipole activation waveform could be modified by varying the trapping waveform frequency because the former is simply a constant fraction of the latter and the latter can be varied, thereby varying the activation frequency [165]. High efficiencies were again observed for reserpine ions ( $\sim 100 \%)$, and a relationship between secular frequency in the DIT and $m / z$ was experimentally derived [166].

\subsection{Ejection}

The most fundamental operation in mass spectrometry is to determine the $\mathrm{m} / \mathrm{z}$ ratios of the ions of interest, that is, to obtain a mass spectrum (a plot of ion intensity vs. $m / z$ ). In order to obtain a mass spectrum, ions must be mass-selectively detected. Detection can be accomplished in quadrupole ion traps in one of two ways: 1) destructive external detection, or 2) nondestructive image charge detection. We begin with the former method, which is by far the most common and certainly much more promising as evidenced by having been commercialized for many years.

Ions are typically ejected from an ion trap by resonance or boundary ejection or a related method and electrostatically focused into a detector, which can be an electron multiplier (low pressure) or a Faraday cup (high pressure). In boundary ejection, also known as the mass selective instability scan, the trapping rf amplitude is ramped linearly with respect to time, thereby moving each ion to the stability "boundary" of the Mathieu stability diagram $(q=0.908)$, at which point ions of that particular $m / z$ are ejected from the trap [83]. Since the $\mathrm{rf}$ amplitude and $m / z$ are 
directly proportional, ions are ejected linearly with time from low to high $m / z$. The signal from the detector is recorded as a function of time, thereby generating an intensity vs. time dataset. Mass calibration, in which compounds of known monoisotopic mass are analyzed in order to linearly correlate time and $m / z$, then yields ion intensity vs. $m / z$, viz. a mass spectrum.

Importantly, the resolution obtained from these mass-selective scan modes is dependent upon the initial conditions of the ions, which will enter the trap with varying $\mathrm{x}, \mathrm{y}$, and $\mathrm{z}$ coordinates and will also have a range of kinetic energies, velocities, and positions. In order to optimize resolution, these parameters must be randomized and the deviation of each reduced. The most common way to accomplish this is by using a process known as collisional cooling $[83,167,168]$. In this method, a light bath/buffer gas such as helium is present in the trap so that collisions between ions and the light neutrals collisionally cool and collapse the ion cloud to the center of the trap, reducing the variation in the initial ion parameters.

The more common method of ion ejection is resonance ejection (Figure 7a) [81, 82], where a small supplementary ac signal is applied across opposite electrodes (e.g. endcaps for the QIT, x electrodes for the LIT) 180 degrees out-ofphase to generate an approximately dipolar field. This scan mode, called the massselective instability scan with resonance ejection, can be illustrated on the Mathieu stability diagram as a "hole" ("black hole") of instability imposed on the q axis (more precisely, instability occurs along an entire iso- $\beta$ line). As in boundary ejection, the rf amplitude is ramped linearly, bringing ions' secular frequencies consecutively into resonance with the supplementary dipolar field such that they pick up energy 
from the driving rf voltage, are excited to higher amplitudes in the trap, and, if the time and amplitude of the ac signal are high enough, the ions are ejected from the trap in order of increasing $m / z$. Resonance ejection offers better performance than mass-selective instability in terms of both resolution and sensitivity due to a more efficient ejection process. Both resonance and boundary ejection of ions can be observed simultaneously if there is a substantial residual population of ions between the resonance point and the boundary (inset, Figure 7a) [169]. These processes can generally be differentiated by their respective peak widths and fundamental substructure.

The choice of both scan rate and scan direction is critical in resonance ejection. Decreasing the scan rate generally increases resolution $[170,171]$, while very fast scanning has the advantage of improving signal intensity [172]. The damping rate must be optimized for the given scan rate, with the resolution being inversely related to the scan rate (ignoring space charge effects) [171]. As stated previously, the forward resonance ejection scan (i.e. scanning the rf amplitude from low to high) gives much better sensitivity and resolution (Figure $\mathbf{4 b}$ ) due to forward ion secular frequency shifts (Figure 4a) [66, 77, 85, 87, 173]. The ideal choice of scan direction, however, depends on the characteristic fields of the ion trap.

The scan direction additionally affects mass assignments due to space charge effects $[94,173]$. In the forward scan, lower mass ions will experience more space charge effects than high mass ions, causing shifts in mass that are particularly noticeable for isotopic distributions. Mass shifts can be reduced by increasing the 
ejection $\mathrm{q}_{\mathrm{u}}$ value, increasing the excitation amplitude, and increasing the scan speed [95]. The usual method of controlling mass shifts to maintain linear mass calibration is a linear ramp of the amplitude of the supplemental ac waveform [174]. However, an interesting application of mass shifts is resolution of isobars and isomers, by taking advantage of the dependence of mass shifts on chemical structure $[175,176]$.

Pressure is another variable that has a significant effect on resonance ejection. While $\sim$ mTorr pressures of bath gas are desirable to increase injection efficiency and mass resolution by collisional cooling [83], in general the buffer gas will broaden mass peaks during mass analysis. It should be noted that very high scan speeds will tend to decrease the effect of buffer gas pressure [177], whereas at low scan speeds there will be an optimal pressure of bath gas (and pressuredependent ac amplitude) which gives maximum resolution $[178,179]$. In general, the equation governing mass resolution $(\Delta \mathrm{m} / \mathrm{m})$ in ion traps is
$\Delta \mathrm{m} / \mathrm{m} \propto \mathrm{P} / \Omega=4 \operatorname{sqrt}(3) / \Omega \tau$
Eq 14

where $P$ is the buffer gas pressure and $\tau$ is the ion motion relaxation time caused by collisions with the bath gas molecules $[180,181]$. Resolution is expected to linearly decrease with bath gas pressure, but this can be countered by operating at higher drive frequency (which, in turn, requires either a smaller trap radius or higher operating voltage).

While ions are generally scanned out via apertures in electrodes (endcaps with holes or x electrodes with slits), unidirectional ion ejection can be 
accomplished by using a resonance ejection waveform that is a linear combination of the nominal dipolar excitation frequency as well as the second harmonic [182]. The direction and efficiency of ejection can be controlled by modifying the ratio of the signal amplitudes. Unidirectional ejection is achieved when the harmonic has the larger amplitude. In modern commercial instruments, ejection direction can be controlled by the phase relationship between the trapping field $\mathrm{RF}$ and the resonance ejection voltage when their frequencies have an integer relationship. In a linear quadrupole ion trap, ions can additionally be scanned out either axially (along the length of the trap) [49] or radially [50,183] through slits in the quadrupole electrodes. These different methods are used in commercial Thermo and Sciex instruments, respectively. The axial ejection method takes advantage of the coupling of ion motion in fringe fields near the ends of the trap (i.e. the "cone of reflection") while the latter relies on excitation along a single axis. Axial ejection can be enhanced with auxiliary electrodes for ion extraction [184, 185], but unlike radial ejection, added octopole fields have not been shown to increase resolution or sensitivity [186].

Resonance ejection is typically performed at a Mathieu $\mathrm{q}_{\mathrm{u}}$ in the vicinity of 0.88 but can also be performed at lower values of $q$ in order to extend the mass range $[187,188]$. However, because ejection is performed away from the optimal $\mathrm{q}_{\mathrm{u}}$ value, resolution and sensitivity are sacrificed in order to achieve this extension. Overtone and sideband frequencies can also be used for mass range extension, though they require higher ac amplitudes because they are fundamentally weaker resonances [189]. 
Resolution can further be improved by performing double or triple resonance ejection. Double resonance ejection has been demonstrated and incorporated into Bruker instruments [190], and it has since been adopted in miniature cylindrical ion traps [181, 191]. In a double resonance ejection scan, the resonance ejection frequency is chosen to match a nonlinear resonance point. These are most commonly the hexapolar resonances at one-third $\left(\beta_{z}=2 / 3\right)$ and one-sixth $\left(\beta_{z}=1 / 3\right)$ the $r f$ frequency or the octopolar nonlinear resonance at one-fourth $\left(\beta_{\mathrm{z}}=1 / 2\right)$ the $\mathrm{rf}$ frequency [192]. The term "double resonance" therefore refers to the co-occurrence of the dipolar resonance and the nonlinear resonance. The coupling of the two resonances is claimed to cause the rate of ion ejection to be faster than the rate encountered in typical single frequency resonance ejection, resulting in superior resolution and sensitivity. An analogous triple resonance ejection mode has been demonstrated and incorporated into Varian instruments [84]. A hexapolar field is intentionally introduced by applying an ac voltage to the endcaps 180 degrees out of phase with respect to the $\mathrm{rf}$ frequency. A dipolar ac frequency is chosen to match a hexapolar resonance and a low-voltage quadrupolar field is then also applied and chosen to match the lower rf sideband $(\Omega-\omega)$ corresponding to the same resonance, thereby generating a triple resonance ejection scan (Figure 7d), which is claimed to give even better resolution than a double resonance (Figure 7c) scan. Care must be taken in both single and multiple frequency resonance ejection experiments to phase-lock the rf and ac waveforms in order to prevent mass peaks from splitting and to maintain mass accuracy $[191,193-195]$. 
Multiple resonance ejection points can be used in compressive mass spectrometry, or multi-resonant frequency excitation ( $\mathrm{mFRE}$ ), illustrated on the Mathieu stability diagram in Figure 7b [196]. In contrast to double and triple resonance ejection, which combine multiple frequencies that correspond to the same Mathieu qu parameter, this method combines frequencies that correspond to different Mathieu qu parameters. As a result, multiple "holes" of instability are imposed on the stability diagram. When the rf amplitude is ramped, multiple sets of ions are detected simultaneously, reducing the time needed for mass analysis by a factor of three to six in the first particular implementation. The signal-to-noise ratio was found to be inferior when using multiple frequencies, and resolution is different for different mass/charge subranges because of the different $\mathrm{q}_{\text {eject }}$ values. In addition, the mass spectra must be "uncompressed." That is, the underlying ion distribution must be calculated from the mFRE data, of which there must be multiple different measurements (i.e. a two-frequency measurement and a threefrequency measurement). Nonetheless, a reconstruction algorithm has been described [196].

Multiple frequency resonance ejection need not take place on a single pair of electrodes. Rhombic excitation and ejection has been implemented in a linear ion trap with simultaneous excitation in $\mathrm{x}$ and $\mathrm{y}$ [197]. Key considerations are the amplitudes and frequencies applied as well as the phase difference between them, particularly if the dimensions in $\mathrm{x}$ and $\mathrm{y}$ are different, which would cause ion secular frequencies in those dimensions to differ. Ion excitation profiles differ based on the phase difference, with a 90-degree phase shift generating rhombic ion motion and a 
zero-degree phase shift generating linear excitation. The key advantage of rhombic excitation and ejection is that as the ions are excited to higher trajectories, they circle the rest of the ion cloud, reducing interactions between the ejected ions and the rest of the ion cloud. This results in a substantial reduction in space charge, which is known to cause peak broadening (compare Figure 7e and f). Space charge can similarly be reduced by using successive resonances for ion ejection [194]. The normal single resonance frequency is replaced by a dual-frequency signal with frequencies spaced very closely (ideally this difference is equivalent to the mass scan rate). When ions encounter the first, lower-amplitude resonance frequency, they are excited to higher trajectories, thereby decreasing space charge induced peak broadening when they are then ejected by the secondhigher-amplitude frequency component. The successive effect can be observed in both the rf amplitude scan and ac frequency scan (discussed later) modes.

Alternative methods of mass-selective ejection can be deduced from the Mathieu parameters $\mathrm{a}_{\mathrm{u}}$ and $\mathrm{q}_{\mathrm{u}}$. The parameters that can be varied in order to accomplish a mass scan are the $\mathrm{rf}$ amplitude $\left(\mathrm{V}_{\mathrm{rf}}\right)$, as already discussed, the DC amplitude (U), the internal radius of the trap ( $\left.\mathrm{r}_{0}\right)$, and the angular $\mathrm{rf}$ frequency $(\Omega)$.

An uncommon method of mass analysis is to scan the rf frequency while holding the rf amplitude constant. This method was first demonstrated by Landais et al. [198] and is the method of choice in digital ion traps $[164,199]$. The attractiveness of this method is the ease with which a frequency can be scanned, especially compared to a linear scan of a high voltage rf. Additionally, mass range can be extended quite easily since the the rf voltage usually limits this range. For rf 
amplitude ramps, the mass range is limited by the maximum $\mathrm{rf}$ voltage that can practically be synthesized and applied without discharges and system instability. Thus, the rf frequency scan has been particularly useful in the analysis of microparticles [200, 201], nanoparticles [202], and large biomolecules [203-205]. According to the Mathieu parameters, mass calibration is nonlinear with a linear frequency sweep, which is a disadvantage of the method. Digital traps, on the other hand, are particularly appropriate devices for frequency scanning because they can modulate the period of the trapping digital waveform [164, 199, 206-208]. Digital traps use high-frequency switching technology to alternate between high-voltage positive (HIGH) and negative (LOW) states, and by varying the ratio of time that the waveform spends on each state (i.e. the duty cycle), a mass spectrum can be recorded in the same manner that rf frequency mass spectra can be recorded. Similarly, resonance ejection can be performed in digital traps by dividing down the trapping digital waveform to obtain a lower-frequency and lower-amplitude dipolar signal [165]. As the trapping waveform duty cycle varies during the mass scan, the supplemental ac frequency will also vary and will be a constant fraction (e.g. 1/3) of the trapping waveform, thereby keeping $q_{\text {eject }}$ constant. More recently it has been demonstrated that resolution may be improved by using a non-integral ratio between the trapping and excitation waveforms, but apparent beat frequencies force ions to be ejected at integer $m / z$ values (assuming the beats line up with integer $m / z$ ), which will corrupt any but the simplest mass spectra [209].

While in theory the radius of the ion trap can be altered during a mass scan with, for example, a stepper motor or linear actuator, it is impossible in practice to 
generate a mass spectrum using this method due to 1) changing electric field components as the internal radius is scanned, and 2) the high precision and accuracy that would be required from the motors. The more promising use of the $r_{0}$ parameter is in ion trap arrays, where traps of different sizes are used to massselect ions of different $m / z$ while using the same set of electronics to drive each trap [210].

Traps generally do not operate with DC components $\left(a_{u}=0\right)$; however, in practice there are some uses for non-zero Mathieu $\mathrm{a}_{\mathrm{u}}$ parameters. As previously mentioned, isolation can be accomplished by using the rf/dc method [115]. However, a mass spectrum can also be recorded by using a DC downscan (capscan), a reverse scan, or an anglescan [211,212], all nonresonance techniques. To effect a reverse scan, the amplitude of the rf is increased to bring higher mass ions to higher $\mathrm{q}_{\mathrm{u}}$ values. A DC potential is then applied so that ions have nonzero Mathieu $\mathrm{a}_{\mathrm{u}}$ parameters. As in a typical quadrupole mass filter, the rf and dc potentials are ramped linearly (but in the reverse direction) keeping their ratio constant, thereby generating a mass spectrum. The DC downscan experiment shows particular promise for analysis of high mass ions since high mass analysis generally requires impractically high $\mathrm{rf}$ amplitudes (or unconventional $\mathrm{rf}$ frequency scanning). With the downscan technique, the rf amplitude is held constant while the dc potential applied to the endcaps (a capscan) or ring electrode (downscan) is ramped to eject ions nonlinearly with time. In the anglescan experiment, which is a variant of the downscan, the rf amplitude is decreased linearly while the dc amplitude is increased. 
Another technique for obtaining a mass spectrum can be deduced from consideration of ion secular frequencies. Though not common, the rf frequency and amplitude can be kept constant while the frequency of the supplementary dipolar ac signal is ramped in a secular frequency scan $[8,114,213]$. This kind of scan may be particularly advantageous for miniature mass spectrometers, which benefit from small, power-efficient electronics. Since the rf amplitude need only be fixed (not ramped linearly in a precise manner), the electronics are simplified. Furthermore, power savings are expected because lower rf amplitudes can be used to achieve higher mass ranges [156]. "Black holes" are a concern in this method, with blank intensity profiles and split peaks at nonlinear resonance points in forward and reverse frequency scans, respectively. Although mass calibration is nonlinear because the relationship between $m / z$ and frequency is not analytically described, a procedure which utilizes the Mathieu parameters $\mathrm{a}_{\mathrm{u}}, \mathrm{q}_{\mathrm{u}}$, and $\beta_{\mathrm{u}}$ showing $<0.1 \%$ calibration error has recently been demonstrated [213]. The calibration method can be modified in order to create a custom frequency-swept waveform to give a linear relationship between $m / z$ and time in a scan termed the "inverse Mathieu q scan" [214]. If the ac frequency is varied so that an inverse relationship exists between the ejected ion's Mathieu qu parameter and time, then the calibration procedure involves performing a linear fit of $m / z$ vs. time, the same procedure that is performed for analytical rf ramp scans. An advantage of the inverse Mathieu q scan is the natural extension of mass range, since typically mass range is limited by the highest voltage that can be achieved by the rf coil without causing a discharge [215]. A further advantage of ac frequency scanning is that, when used as a method of 
mass-selective excitation and combined with a fixed frequency component set on a particular product ion, a precursor scan can be performed as a single scan using a single ion trap. This was first demonstrated by Yost [157] but has since been demonstrated on a miniature mass spectrometer [158]. Whether the ac frequency scan is comparable to the rf amplitude scan in terms of simplicity, scan rate, mass accuracy, and resolution have yet to be determined. For example, the amplitude of the ac has so far been kept constant, but one would expect this amplitude to vary with $m / z$ because of potential well depth (as is the case with resonance ejection [174]).

So far we have discussed methods of scanning ions out of a quadrupole ion trap, but for targeted applications the entire ion population need not be detected. Instead, the trap could be operated in a non-scanning mode. For example, a nonscanning rectilinear ion trap for selected ion and selected reaction monitoring has been described [216]. Ions were injected into the RIT and a DC offset on the y rods was used for apex isolation of a particular precursor ion, which could then be ejected axially by applying a DC pulse on one of the axial z electrodes. Thus, the intensity of a single $m / z$ species is monitored, and a full scan mass spectrum cannot be collected unless the experiment is repeated for each $m / z$. A similar experiment was implemented on a linear ion trap made using digital light processing technology [217], and it has recently been calculated that a mass resolution of 1 amu should be possible in the non-scanning mode [218]. 


\subsection{Charge detection}

Image charge detection of trapped ions can be classified into two types. The first type is to measure the ion image charge (or resulting induced voltage) with a charge detector coupled with the ion trap, e.g. gated electrostatic ion trap $[219,220]$ or a quadrupole ion trap [221, 222]. Conventional secondary electron multiplier detectors are not able to generate enough signal at low ion velocity because of low secondary electron yield; a charge detector, however, is independent of ion velocity, so it can overcome the ion detection problem of macroions like viruses and cells [223]. The second approach is to measure the ion image current by connecting a differential amplifier circuit to a pair of electrodes in the quadrupole ion trap [224], Fourier transform ion cyclotron resonance (FTICR) [225] or Orbitrap mass spectrometers [226], which we briefly discuss due to the similarities between the detection schemes between these instruments an charge detection QITs. With a Fourier transform of ion signals, high resolving power is obtained, but in the case of the ion trap great care must be taken to observe the small ion signal in the presence of the very large rf signal [227].

Gated electrostatic ion traps can measure the charge and mass-to-charge ratios of large electrosprayed ions nondestructively as shown in Figure 8a [219, 228]. In Figure 8b, by repeatedly measuring the ions within the electrostatic ion trap, the uncertainty of the charge measurement could be reduced to $\sim 0.2$ e [220]. In Figure 8c, a quadrupole ion trap coupled with a charge detector was used to nondestructively measure the charge and mass-to-charge ratios of laser-induced acoustic desorbed macroions [221, 222, 229]. 
The mass resolution of charge-detection quadrupole ion trap instruments depends on the measurement accuracy, the mass-to-charge ratios, and also charge. The coefficient of variance of the measured individual particles' ejection points ( $\mathrm{q}_{\mathrm{eject}}$ ) at high pressure (40 mtorr) is about $1.2 \%$, ensuring the mass resolution is approximately 100 [230]. The accuracy in charge measurement depends on the number of charges carried by individual particles [231].

The use of ion traps with image charge (or induced current) detection circuits can result in high resolving power and mass accuracy. Guan and Marshall proposed stored waveform inverse Fourier transform (SWIFT) for broadband massselective excitation and ejection of ions in quadrupole ion trap and FT-ICR mass spectrometers [124, 232, 233]. Figure 9a shows that non-destructive ion detection with a broad-band Fourier transform quadrupole ion trap can reach a resolution of $\sim 1000$ at a detection bandwidth of $400 \mathrm{kHz}[224,234]$. Figure $9 \mathbf{b}$ shows the method of trapping, excitation, and detection of ions in FT-ICR cells [225]. Unit mass resolution of high mass molecules (chondroitinase, $112 \mathrm{kDa}$ ) with 3 Da mass accuracy has been achieved with a 9.4 tesla FT-ICR mass spectrometer [235]. Recently, a 21 tesla FT-ICR mass spectrometer was developed and took advantage of the linear quadrupole trap for high ion capacity and ejection efficiency; a mass resolving power of $150,000(\mathrm{~m} / \Delta \mathrm{m}, 50 \%)$ was achieved for bovine serum albumin ( $66 \mathrm{kDa}$ ) for a $0.38 \mathrm{~s}$ detection period, and $>2,000,000$ resolving power is achieved for a $12 \mathrm{~s}$ detection period. However, the cost to maintain the operation of an FT-ICR instrument is high. To reduce the cost, an electrostatic ion trap device, i.e. Orbitrap [226, 236-238] or Cassinian trap [239, 240] were developed. 


\section{Conclusion}

The quadrupole ion trap has a storied history of development over the past six and a half decades, becoming one of the most sensitive, selective, and versatile analytical second order trap, shows resolving powers of 53,000 for the fundamental frequency and 140,000 for the third harmonic in $1 \mathrm{~s}$. Broad-band and narrow-band waveforms are used to de-excite/re-excite ion motion and unit mass selection is achieved by selective de-excitation and re-excitation with narrow-band waveforms [241]. In addition, phase-enhanced selective ion ejection based on broadband dipolar excitation and ion ejection shows an isolation resolution of 28,400 and a mass resolution of 100,000 with an Orbitrap [242]. By rephasing the ion packet with a narrower axial spatial extent and frequency distribution in an Oribitrap, mass resolutions of 124,000 to 171,000 are obtained which is a 2 - to 3 -fold improvement over the resolution of 40,000 to 60,000 without rephasing [243].

High mass ions, e.g. GroEL and immunoglobulin G (IgG) can be detected by implementing ion optics for ion transport with ion funnels and quadrupoles and extending software detection mass range to $\sim \mathrm{m} / \mathrm{z} 20,000$ [244, 245]. MS/MS of high mass ions with an Orbitrap device is arranged in tandem-in-space method, i.e. collisional-induced fragmentation at high pressure conditions in collision cells. $\mathrm{MS}^{3}$ is demonstrated with an Orbitrap instrument by analyzing the GroEL fragment ions and a mass measurement accuracy of $2.3 \mathrm{ppm}$ is achieved [244]. 
devices. While complications arise from the co-occurrence of linear and higherorder field resonances, they have nonetheless proved quite useful for increasing the performance and overall versatility of these already-advantageous devices. With new developments in multi-trap systems, QIT-FT hybrids (e.g. QIT-Orbitrap), frequency scanning, digital ion trap technology, ion trap miniaturization and multiplexing on the horizon, it will become increasingly important to further understand ion trap resonances in order to continue to exploit them for optimized performance.

\section{Acknowledgements}

The authors acknowledge funding from NSF (CHE 1307264) and NASA (NNX16AJ25G) and recognize outstanding contributions from many fellow scientists. The authors also gratefully acknowledge the contributions of Jae Schwartz (Thermo Fisher Scientific), who reviewed the article prior to submission. WPP thanks the support by grants from the Ministry of Science and Technology of Taiwan (MOST 102-2112-M-259-003-MY3 and 105-2918-I-259-001) and appreciates Prof. R.G. Cooks for the opportunity to visit his lab. 
biologically active molecules onto surfaces. His group is also responsible for the ambient ionization method desorption electrospray ionization (DESI). Most recently his interests have focused on applications of mass spectrometry to chemical synthesis in microdroplets, intrasurgical diagnostics, and origin-of-life chemistry. Cooks has authored 1,100 publications and served as thesis advisor to $130 \mathrm{Ph}$. D.'s.

\section{References}

[1] R.D. Espy, M. Wleklinski, X. Yan, R.G. Cooks, Beyond the flask: Reactions on the fly in ambient mass spectrometry, TrAC Trends in Analytical Chemistry, 57 (2014) 135-146.

[2] A. Colorado, D.J. Barket, J.M. Hurst, P.B. Shepson, A fast-response method for determination of atmospheric isoprene using quadrupole ion trap mass spectrometry, Analytical Chemistry, 70 (1998) 5129-5135.

[3] L.H. Mielke, K.A. Pratt, P.B. Shepson, S.A. McLuckey, A. Wisthaler, A. Hansel, Quantitative determination of biogenic volatile organic compounds in the atmosphere using proton-transfer reaction linear ion trap mass spectrometry, Anal Chem, 82 (2010) 7952-7957.

[4] W. Paul, H. Steinwedel, A new mass spectrometer without a magnetic field, Z. Naturforsch. Sect. A, 8 (1953) 448-450.

[5] W. Paul, Electromagnetic traps for charged and neutral particles, Reviews of modern physics, 62 (1990) 531.

[6] M. Welling, R.I. Thompson, H. Walther, Photodissociation of MgC60+ complexes generated and stored in a linear ion trap, Chemical Physics Letters, 253 (1996) 3742.

[7] N. Mikami, Y. Miyata, S. Sato, T. Sasaki, ION TRAP METHOD COMBINED WITH TWO-COLOR LASER SPECTROSCOPY OF SUPERSONIC MOLECULAR BEAMSPHOTODISSOCIATION OF TRAPPED C6H5Cl+, Chemical Physics Letters, 166 (1990) 470-474.

[8] M. Welling, H.A. Schuessler, R.I. Thompson, H. Walther, Ion/molecule reactions, mass spectrometry and optical spectroscopy in a linear ion trap, Int. J. Mass Spectrom. Ion Processes, 172 (1998) 95-114.

[9] S. Pollack, D. Cameron, M. Rokni, W. Hill, J.H. Parks, Charge exchange and cluster formation in an rf Paul trap: interaction of alkali atoms with C60+, Chemical Physics Letters, 256 (1996) 101-108.

[10] H. Hiura, T. Kanayama, Growth of hydrogenated silicon cluster ions using an ion trap, Chemical Physics Letters, 328 (2000) 409-414.

[11] E.R. Lovejoy, R. Bianco, Temperature dependence of cluster ion decomposition in a quadrupole ion trap, Journal of Physical Chemistry A, 104 (2000) 10280-10287. 
[12] Y.S. Wang, C.H. Tsai, Y.T. Lee, H.C. Chang, J.C. Jiang, O. Asvany, S. Schlemmer, D. Gerlich, Investigations of protonated and deprotonated water clusters using a lowtemperature 22-pole ion trap, Journal of Physical Chemistry A, 107 (2003) 42174225.

[13] P.H. Hemberger, N.S. Nogar, J.D. Williams, R.G. Cooks, J.E.P. Syka, Laser Photodissociation Probe for Ion Tomography Studies in a Quadrupole Ion-Trap Mass-Spectrometer, Chemical Physics Letters, 191 (1992) 405-410.

[14] C.D. Cleven, R.G. Cooks, A.W. Garrett, N.S. Nogar, P.H. Hemberger, Radial distributions and ejection times of molecular ions in an ion trap mass spectrometer:

A laser tomography study of effects of ion density and molecular type, Journal of Physical Chemistry, 100 (1996) 40-46.

[15] A. Steane, The ion trap quantum information processor, Applied Physics BLasers and Optics, 64 (1997) 623-642.

[16] G.J. Milburn, S. Schneider, D.F.V. James, Ion trap quantum computing with warm ions, Fortschritte Der Physik-Progress of Physics, 48 (2000) 801-810.

[17] D. Kielpinski, C. Monroe, D.J. Wineland, Architecture for a large-scale ion-trap quantum computer, Nature, 417 (2002) 709-711.

[18] S. Gulde, M. Riebe, G.P. Lancaster, C. Becher, J. Eschner, H. Haffner, F. SchmidtKaler, I.L. Chuang, R. Blatt, Implementation of the Deutsch-Jozsa algorithm on an ion-trap quantum computer, Nature, 421 (2003) 48-50.

[19] D. Porras, J.I. Cirac, Effective quantum spin systems with trapped ions, Phys Rev Lett, 92 (2004) 207901.

[20] S. Seidelin, J. Chiaverini, R. Reichle, J.J. Bollinger, D. Leibfried, J. Britton, J.H. Wesenberg, R.B. Blakestad, R.J. Epstein, D.B. Hume, W.M. Itano, J.D. Jost, C. Langer, R. Ozeri, N. Shiga, D.J. Wineland, Microfabricated surface-electrode ion trap for scalable quantum information processing, Phys Rev Lett, 96 (2006) 253003.

[21] J.P. Home, D. Hanneke, J.D. Jost, J.M. Amini, D. Leibfried, D.J. Wineland, Complete methods set for scalable ion trap quantum information processing, Science, 325 (2009) 1227-1230.

[22] C. Monroe, J. Kim, Scaling the ion trap quantum processor, Science, 339 (2013) 1164-1169.

[23] D.J. Wineland, J.C. Bergquist, W.M. Itano, J.J. Bollinger, C.H. Manney, Atomic ion Coulomb clusters in an ion trap, Phys Rev Lett, 59 (1987) 2935-2938.

[24] L. Hornekaer, N. Kjaergaard, A.M. Thommesen, M. Drewsen, Structural properties of two-component coulomb crystals in linear paul traps, Phys Rev Lett, 86 (2001) 1994-1997.

[25] D. Porras, J.I. Cirac, Quantum manipulation of trapped ions in two dimensional coulomb crystals, Phys Rev Lett, 96 (2006) 250501.

[26] L. Schmoger, 0.O. Versolato, M. Schwarz, M. Kohnen, A. Windberger, B. Piest, S. Feuchtenbeiner, J. Pedregosa-Gutierrez, T. Leopold, P. Micke, A.K. Hansen, T.M. Baumann, M. Drewsen, J. Ullrich, P.O. Schmidt, J.R. Lopez-Urrutia, Coulomb crystallization of highly charged ions, Science, 347 (2015) 1233-1236.

[27] R. Schinke, Photodissociation Dynamics: Spectroscopy and Fragmentation of Small Polyatomic Molecules, Cambridge University Press, New York, 1993. 
[28] Y. Inokuchi, O.V. Boyarkin, R. Kusaka, T. Haino, T. Ebata, T.R. Rizzo, Ion selectivity of crown ethers investigated by UV and IR spectroscopy in a cold ion trap, J Phys Chem A, 116 (2012) 4057-4068.

[29] S. Gartner, J. Krieg, A. Klemann, O. Asvany, S. Brunken, S. Schlemmer, Highresolution spectroscopy of $\mathrm{CH} 2 \mathrm{D}+$ in a cold 22-pole ion trap, J Phys Chem A, 117 (2013) 9975-9984.

[30] J.G. Redwine, Z.A. Davis, N.L. Burke, R.A. Oglesbee, S.A. McLuckey, T.S. Zwier, A novel ion trap based tandem mass spectrometer for the spectroscopic study of cold gas phase polyatomic ions, International Journal of Mass Spectrometry, 348 (2013) 9-14.

[31] N.L. Burke, J.G. Redwine, J.C. Dean, S.A. McLuckey, T.S. Zwier, UV and IR spectroscopy of cold protonated leucine enkephalin, International Journal of Mass Spectrometry, 378 (2015) 196-205.

[32] N.L. Burke, A.F. DeBlase, J.G. Redwine, J.R. Hopkins, S.A. McLuckey, T.S. Zwier, Gas-Phase Folding of a Prototypical Protonated Pentapeptide: Spectroscopic Evidence for Formation of a Charge-Stabilized beta-Hairpin, J Am Chem Soc, 138 (2016) 2849-2857.

[33] D.A. McQuarrie, J.D. Simon, Physical chemistry: A molecular approach, 1997. [34] J.S. Brodbelt, Photodissociation mass spectrometry: new tools for characterization of biological molecules, Chem. Soc. Rev., 43 (2014) 2757-2783. [35] R.L. Mössbauer, Kernresonanzfluoreszenz von Gammastrahlung in Ir191, Zeitschrift für Physik, 151 (1958) 124-143.

[36] E.R. Andrew, Nuclear magnetic resonance, Nuclear Magnetic Resonance, by ER Andrew, Cambridge, UK: Cambridge University Press, 2009, 1 (2009).

[37] N.M. Atherton, M. Davies, B. Gilbert, Electron spin resonance, Royal Society of Chemistry, 1994.

[38] W.B. Lynch, K.A. Earle, J.H. Freed, 1-mm wave ESR spectrometer, Review of scientific instruments, 59 (1988) 1345-1351.

[39] A.P. Seyranian, The swing: Parametric resonance, Journal of Applied Mathematics and Mechanics, 68 (2004) 757-764.

[40] R.C. Eckardt, C.D. Nabors, W.J. Kozlovsky, R.L. Byer, Optical parametric oscillator frequency tuning and control, J. Opt. Soc. Am. B, 8 (1991) 646-667. [41] R.E. March, J.F.J. Todd, Quadrupole ion trap mass spectrometry, WileyInterscience, 2005.

[42] P.H. Dawson, Quadrupole mass spectrometry and its applications, Elsevier, 2013.

[43] R.D. Knight, The general form of the quadrupole ion trap potential, International Journal of Mass Spectrometry and Ion Physics, 51 (1983) 127-131. [44] E. Mathieu, Math. Pure Appl. (J. Liouville), 13 (1868) 137.

[45] H.G. Dehmelt, Radiofrequency spectroscopy of stored ions I: Storage, Adv. At. Mol. Phys., 3 (1968) 53-72.

[46] D.J. Douglas, A.J. Frank, D. Mao, Linear ion traps in mass spectrometry, Mass Spectrom. Rev., 24 (2005) 1-29.

[47] P.T.A. Reilly, G.F. Brabeck, Mapping the pseudopotential well for all values of the Mathieu parameter q in digital and sinusoidal ion traps, Int. J. Mass Spectrom., 392 (2015) 86-90. 
[48] C. Gao, D.J. Douglas, Can the effective potential of a linear quadrupole be extended to values of the Mathieu parameter q up to 0.90?, J Am Soc Mass Spectrom, 24 (2013) 1848-1852.

[49] J.C. Schwartz, M.W. Senko, J.E. Syka, A two-dimensional quadrupole ion trap mass spectrometer, J Am Soc Mass Spectr, 13 (2002) 659-669.

[50] J.W. Hager, A new linear ion trap mass spectrometer, Rapid Commun Mass Sp, 16 (2002) 512-526.

[51] M.E. Bier, J.E.P. Syka, Ion trap mass spectrometer system and method, in, Finnigan Corporation, United States, 1995.

[52] E.R. Badman, R. Graham Cooks, Miniature mass analyzers, J. Mass Spectrom., 35 (2000) 659-671.

[53] J.E. Fulford, R.E. March, R.E. Mather, J.F.J. Todd, R.M. Waldren, The cylindrical ion trap - a theoretical and experimental study, Can. J. Spectrosc., 25 (1980) 85-97.

[54] E.R. Badman, R.C. Johnson, W.R. Plass, R.G. Cooks, A miniature cylindrical quadrupole ion trap: simulation and experiment, Anal. Chem., 70 (1998) 4896-4901.

[55] J.M. Wells, E.R. Badman, R.G. Cooks, A quadrupole ion trap with cylindrical geometry operated in the mass-selective instability mode, Anal. Chem., 70 (1998) 438-444.

[56] G.E. Patterson, A.J. Guymon, L.S. Riter, M. Everly, J. Griep-Raming, B.C. Laughlin, Z. Ouyang, R.G. Cooks, Miniature Cylindrical Ion Trap Mass Spectrometer, Anal. Chem., 74 (2002) 6145-6153.

[57] W.-W. Lee, C.-H. Oh, P.-S. Kim, M. Yang, K. Song, Characteristics of cylindrical ion trap, International Journal of Mass Spectrometry, 230 (2003) 25-31.

[58] Z. Ouyang, G. Wu, Y. Song, H. Li, W.R. Plass, R.G. Cooks, Rectilinear ion trap: concepts, calculations, and analytical performance of a new mass analyzer, Anal. Chem., 76 (2004) 4595-4605.

[59] S.A. Lammert, W.R. Plass, C.V. Thompson, M.B. Wise, Design, optimization and initial performance of a toroidal $r$ ion trap mass spectrometer, Int. J. Mass Spectrom., 212 (2001) 25-40.

[60] S.A. Lammert, A.A. Rockwood, M. Wang, M.L. Lee, E.D. Lee, S.E. Tolley, J.R. Oliphant, J.L. Jones, R.W. Waite, Miniature toroidal radio frequency ion trap mass analyzer, J Am Soc Mass Spectr, 17 (2006) 916-922.

[61] N. Taylor, D.E. Austin, A simplified toroidal ion trap mass analyzer, Int. J. Mass Spectrom., 321-322 (2012) 25-32.

[62] J.M. Higgs, D.E. Austin, Simulations of ion motion in toroidal ion traps, International Journal of Mass Spectrometry, 363 (2014) 40-51.

[63] D.E. Austin, M. Wang, S.E. Tolley, J.D. Maas, A.R. Hawkins, A.L. Rockwood, H.D. Tolley, E.D. Lee, M.L. Lee, Halo ion trap mass spectrometer, Anal. Chem., 79 (2007) 2927-2932.

[64] B.A. Collings, D.J. Douglas, Observation of higher order quadrupole excitation frequencies in a linear ion trap, J Am Soc Mass Spectr, 11 (2000) 1016-1022. [65] B.A. Collings, M. Sudakov, F.A. Londry, Resonance shifts in the excitation of the $\mathrm{n}=0, \mathrm{~K}=1$ to 6 quadrupolar resonances for ions confined in a linear ion trap, $\mathrm{J}$ Am Soc Mass Spectr, 13 (2002) 577-586.

[66] J. Franzen, The non-linear ion trap. Part 5. Nature of non-linear resonances and resonant ion ejection, Int. J. Mass Spectrom. Ion Processes, 130 (1994) 15-40. 
[67] M. Sudakov, Effective potential and the ion axial beat motion near the boundary of the first stable region in a nonlinear ion trap, Int. J. Mass Spectrom., 206 (2001) 27-43.

[68] Q. Wu, Y. Tian, A. Li, D.E. Austin, Simulations of electrode misalignment effects in two-plate linear ion traps, International Journal of Mass Spectrometry, 393 (2015) 52-57.

[69] W. Xu, W.J. Chappell, R.G. Cooks, Z. Ouyang, Characterization of electrode surface roughness and its impact on ion trap mass analysis, J Mass Spectrom, 44 (2009) 353-360.

[70] X. Huo, F. Tang, J. Chen, X. Zhang, X. Wang, Characterization of the impact of the ejection slit on miniature rectilinear ion trap analysis, International Journal of Mass Spectrometry, 399-400 (2016) 44-50.

[71] M. Chattopadhyay, A.K. Mohanty, Approximate multipole coefficients of RF ion traps as functions of aperture size, International Journal of Mass Spectrometry, 295 (2010) 49-59.

[72] The non-linear ion trap. Part 3. Multipole components in three types of practical ion trap.

[73] P.H. Dawson, N.R. Whetten, Non-linear resonances in quadrupole mass spectrometers due to imperfect fields. II. the quadrupole mass filter and the monopole mass spectrometer, Int. J. Mass Spectrom. Ion Phys., 3 (1969) 1-12. [74] P.H. Dawson, N.R. Whetten, Non-linear resonances in quadrupole mass spectrometers due to imperfect fields I. The quadrupole ion trap, Int. J. Mass

Spectrom. Ion Phys., 2 (1969) 45-59.

[75] W.R. Plass, Theory of dipolar dc excitation and dc tomography in the rf quadrupole ion trap, Int. J. Mass Spectrom., 202 (2000) 175-197.

[76] C. Weil, J.M. Wells, H. Wollnik, R.G. Cooks, Axial ion motion within the quadrupole ion trap elucidated by dc pulse tomography, International Journal of

Mass Spectrometry, 194 (2000) 225-234.

[77] Y. Wang, Z. Huang, Y. Jiang, X. Xiong, Y. Deng, X. Fang, W. Xu, The coupling effects of hexapole and octopole fields in quadrupole ion traps: a theoretical study, J. Mass Spectrom., 48 (2013) 937-944.

[78] S. Sevugarajan, A.G. Menon, Field imperfection induced axial secular frequency shifts in nonlinear ion traps, International Journal of Mass Spectrometry, 189 (1999) 53-61.

[79] X. Zhou, C. Xiong, S. Zhang, N. Zhang, Z. Nie, Study of nonlinear resonance effect in Paul trap, J Am Soc Mass Spectrom, 24 (2013) 794-800.

[80] C.Q. Xiong, X.Y. Zhou, N. Zhang, L.P. Zhan, Y.T. Chen, Z.X. Nie, Nonlinear Ion Harmonics in the Paul Trap with Added Octopole Field: Theoretical Characterization and New Insight into Nonlinear Resonance Effect, Journal of the American Society for Mass Spectrometry, 27 (2016) 344-351.

[81] J.E. Fulford, D. Nhu-Hoa, R.J. Hughes, R.E. March, R.F. Bonner, G.J. Wong, Radiofrequency mass selective excitation and resonant ejection of ions in a threedimensional quadrupole ion trap, J. Vac. Sci. Technol., 17 (1980) 829-835. [82] J.N. Louris, R.G. Cooks, J.E.P. Syka, P.E. Kelley, G.C. Stafford, J.F.J. Todd, Instrumentation, applications, and energy deposition in quadrupole ion-trap tandem mass-spectrometry, Anal. Chem., 59 (1987) 1677-1685. 
[83] G.C. Stafford, P.E. Kelley, J.E.P. Syka, W.E. Reynolds, J.F.J. Todd, Recent improvements in and analytical applications of advanced ion trap technology, Int. J. Mass Spectrom. Ion Proc., 60 (1984) 85-98.

[84] M. Splendore, E. Marquette, J. Oppenheimer, C. Huston, G. Wells, A new ion ejection method employing an asymmetric trapping field to improve the mass scanning performance of an electrodynamic ion trap, Int. J. Mass Spectrom., 191 (1999) 129-143.

[85] A.A. Makarov, Resonance ejection from the Paul trap: a theoretical treatment incorporating a weak octapole field, Anal. Chem., 68 (1996) 4257-4263.

[86] S. Sevugarajan, A.G. Menon, Frequency perturbation in nonlinear Paul traps: a simulation study of the effect of geometric aberration, space charge, dipolar excitation, and damping on ion axial secular frequency, International Journal of Mass Spectrometry, 197 (2000) 263-278.

[87] J.D. Williams, K.A. Cox, R.G. Cooks, S.A. Mcluckey, K.J. Hart, D.E. Goeringer, Resonance ejection ion-trap mass-spectrometry and nonlinear field contributions the effect of scan direction on mass resolution, Anal. Chem., 66 (1994) 725-729. [88] X.-G. Wu, Ion dynamics in non-perfect quadrupole traps, International Journal of Mass Spectrometry, 263 (2007) 59-65.

[89] Q. Dang, F. Xu, X. Huang, X. Fang, R. Wang, C.F. Ding, Linear ion trap with added octopole field component: the property and method, J Mass Spectrom, 50 (2015) 1400-1408.

[90] Y. Wang, X. Zhang, Y. Feng, R. Shao, X. Xiong, X. Fang, Y. Deng, W. Xu, Characterization of geometry deviation effects on ion trap mass analysis: A comparison study, International Journal of Mass Spectrometry, 370 (2014) 125-131.

[91] J.M. Wells, W.R. Plass, G.E. Patterson, Z. Ouyang, E.R. Badman, R.G. Cooks, Chemical Mass Shifts in Ion Trap Mass Spectrometry: Experiments and Simulations, Anal. Chem., 71 (1999) 3405-3415.

[92] J.M. Wells, W.A. Plass, R.G. Cooks, Control of chemical mass shifts in the quadrupole ion trap through selection of resonance ejection working point and $\mathrm{rf}$ scan direction, Anal. Chem., 72 (2000) 2677-2683.

[93] D. Guo, Y. Wang, X. Xiong, H. Zhang, X. Zhang, T. Yuan, X. Fang, W. Xu, Space charge induced nonlinear effects in quadrupole ion traps, J Am Soc Mass Spectrom, 25 (2014) 498-508.

[94] K.A. Cox, C.D. Cleven, R.G. Cooks, Mass shifts and local space-charge effects observed in the quadrupole ion-trap at higher resolution, Int. J. Mass Spectrom. Ion Proc., 144 (1995) 47-65.

[95] H. Qiao, C. Gao, D. Mao, N. Konenkov, D.J. Douglas, Space-charge effects with mass-selective axial ejection from a linear quadrupole ion trap, Rapid Commun Mass Sp, 25 (2011) 3509-3520.

[96] P. Mandal, S. Das, D. De Munshi, T. Dutta, M. Mukherjee, Space charge and collective oscillation of ion cloud in a linear Paul trap, International Journal of Mass Spectrometry, 364 (2014) 16-20.

[97] M.J. Charles, S.A. Mcluckey, G.L. Glish, Competition between Resonance Ejection and Ion Dissociation during Resonant Excitation in a Quadrupole Ion-Trap, Journal of the American Society for Mass Spectrometry, 5 (1994) 1031-1041. 
[98] M.A. Mabud, M.J. Dekrey, R.G. Cooks, Surface-induced dissociation of molecular ions, Int. J. Mass Spectrom. Ion Processes, 67 (1985) 285-294.

[99] J.E. Syka, J.J. Coon, M.J. Schroeder, J. Shabanowitz, D.F. Hunt, Peptide and protein sequence analysis by electron transfer dissociation mass spectrometry, Proceedings of the National Academy of Sciences of the United States of America, 101 (2004) 9528-9533.

[100] J.C. Schwartz, High-q Pulsed Fragmentation In Ion Traps, in: U. States (Ed.), Thermo Finnigan LLC, United States, 2005.

[101] W.D. Bowers, S.S. Delbert, R.L. Hunter, R.T. McIver, Fragmentation of oligopeptide ions using ultraviolet laser radiation and Fourier transform mass spectrometry, Journal of the American Chemical Society, 106 (1984) 7288-7289. [102] D.P. Little, J.P. Speir, M.W. Senko, P.B. O'Connor, F.W. McLafferty, Infrared Multiphoton Dissociation of Large Multiply Charged Ions for Biomolecule Sequencing, Analytical Chemistry, 66 (1994) 2809-2815. [103] B.A. Collings, W.R. Stott, F.A. Londry, Resonant excitation in a low-pressure linear ion trap, Journal of the American Society for Mass Spectrometry, 14 (2003) 622-634.

[104] A.G. Baker, A. Alexander, M.V. Novotny, Narrow-band collisional activation technique for ion trap mass spectrometers, Anal Chem, 71 (1999) 2945-2950. [105] J.W. Hager, Off-resonance excitation in a linear ion trap, J Am Soc Mass Spectr, 20 (2009) 443-450.

[106] R. Alheit, S. Kleineidam, F. Vedel, M. Vedel, G. Werth, Higher order non-linear resonances in a Paul trap, Int. J. Mass Spectrom. Ion Processes, 154 (1996) 155-169. [107] X.Z. Chu, M. Holzki, R. Alheit, G. Werth, Observation of high-order motional resonances of an ion cloud in a Paul trap, Int. J. Mass Spectrom. Ion Processes, 173 (1998) 107-112.

[108] J.C. Schwartz, J.N. Louris, Method of detecting ions in an ion trap mass spectrometer, in, Google Patents, 1994.

[109] D.M. Eades, R.A. Yost, Black Canyons for Ions Stored in an Ion-trap Mass Spectrometer, Rapid Commun Mass Sp, 6 (1992) 573-578.

[110] F. Guidugli, P. Traldi, A Phenomenological Description of a Black Hole for Collisionally Induced Decomposition Products in Ion-trap Mass Spectrometry, Rapid Commun Mass Sp, 5 (1991) 343-348.

[111] K.L. Morand, S.A. Lammert, R.G. Cooks, Concerning 'Black Holes' in Ion-trap Mass Spectrometry, Rapid Commun Mass Sp, 5 (2005) 491.

[112] D.M. Eades, J.V. Johnson, R.A. Yost, Nonlinear resonance effects during ion storage in a quadrupole ion trap, J Am Soc Mass Spectr, 4 (1993) 917-929.

[113] V.M. Doroshenko, R.J. Cotter, Losses of Ions During Forward and Reverse Scans in a Quadrupole Ion Trap Mass Spectrometer and How to Reduce Them, J Am Soc Mass Spectr, 8 (1997) 1141-1146.

[114] D.T. Snyder, C.J. Pulliam, J.S. Wiley, J. Duncan, R.G. Cooks, Experimental characterization of secular frequency scanning in ion trap mass spectrometers, J Am Soc Mass Spectr, 27 (2016) 1243-1255.

[115] S.A. McLuckey, D.E. Goeringer, G.L. Glish, Selective ion isolation/rejection over a broad mass range in the quadrupole ion trap, J Am Soc Mass Spectr, 2 (1991) 11-

21. 
[116] J.E. Fulford, R.E. March, A new mode of operation for the three-dimensional quadrupole ion store (QUISTOR): The selective ion reactor, Int. J. Mass Spectrom. Ion Phys., 26 (1978) 155-162.

[117] P.E. Kelley, J.E.P. Syka, P.C. Ceja, G.C. Stafford, J.N. Louris, H.F. Grutzmacher, D. Kuck, J.F.J. Todd, in: Proceedings of the 34th Conference on Mass Spectrometry and Allied Topics, Cincinnati, 1986, pp. 963.

[118] R.E. Kaiser Jr., R.G. Cooks, J.E.P. Syka, G.C. Stafford Jr., Collisionally Activated Dissociation of Peptides Using a Quadrupole Ion-Trap Mass Spectrometer, Rapid Commun Mass Sp, 4 (1990) 30-33.

[119] J.C. Schwartz, I. Jardine, High Resolution Parent-ion Selection/Isolation Using a Quadrupole Ion-trap Mass Spectrometer, Rapid Commun Mass Sp, 6 (1992) 313317.

[120] F. Vedel, M. Vedel, R.E. March, New schemes for resonant ejection in r.f. quadrupolar ion traps, Int. J. Mass Spectrom. Ion Processes, 99 (1990) 125-138.

[121] D.T. Snyder, R.G. Cooks, Ion isolation and multigenerational collision-induced dissociation using the inverse Mathieu q scan.

[122] F. Vedel, M. Vedel, R.E. March, A sensitive method for the detection of stored ions by resonant ejection using a wide-band signal, Int. J. Mass Spectrom. Ion Processes, 108 (1991) R11-R20.

[123] L. Chen, A.G. Marchall, Stored waveform simultaneous mass-selective ejection/excitation for Fourier transform ion cyclotron resonance mass spectrometry, Int. J. Mass Spectrom. Ion. Processes, 79 (1987) 115-125. [124] S. Guan, General phase modulation method for stored waveform inverse Fourier transform excitation for Fourier transform ion cyclotron resonance mass spectrometry, J. Chem. Phys., 91 (1989) 775-777.

[125] S. Guan, A.G. Marshall, Stored waveform inverse Fourier transform axial excitation/ejection for quadrupole ion trap mass spectrometry, Anal. Chem., 65 (1993) 1288-1294.

[126] J. Julian, R. K., R.G. Cooks, Broad-Band Excitation in the Quadrupole Ion Trap Mass Spectrometer Using Shaped Pulses Created with the Inverse Fourier Transform, Anal. Chem., 65 (1993) 1827-1833.

[127] M.H. Soni, R.G. Cooks, Selective Injection and Isolation of Ions in Quadrupole Ion Trap Mass Spectrometry Using Notched Waveforms Created Using the Inverse Fourier Transform, Anal. Chem., 66 (1994) 2488-2496.

[128] D.T. Snyder, R.G. Cooks, Ion isolation in a linear ion trap using dual resonance frequencies, J Am Soc Mass Spectr.

[129] J.N. Louris, D.M. Taylor, Method and apparatus for ejecting unwanted ions in an ion trap mass spectrometer, in, Google Patents, 1994.

[130] R.T. Hilger, R.E. Santini, C.A. Luongo, B.M. Prentice, S.A. McLuckey, A method for isolating ions in quadrupole ion traps using an excitation waveform generated by frequency modulation and mixing, Int. J. Mass Spectrom., 377 (2015) 329-337. [131] D.V. Kenny, P.J. Callahan, S.M. Gordon, S.W. Stiller, Simultaneous Isolation of Two Different $\mathrm{m} / \mathrm{z}$ Ions in an Ion-trap Mass Spectrometer and their Tandem Mass Spectra Using Filtered-noise Fields, Rapid Commun Mass Sp, 7 (1993). 
[132] D.E. Goeringer, K.G. Asano, S.A. Mcluckey, D. Hoekman, S.W. Stiller, Filtered Noise Field Signals for Mass-Selective Accumulation of Externally Formed Ions in a Quadrupole Ion-Trap, Analytical Chemistry, 66 (1994) 313-318.

[133] K. Miseki, Ion trap mass spectrometer, in: U. States (Ed.), Shimadzu Corporation, United States, 2002.

[134] G. Wells, C. Huston, High-Resolution Selected-Ion Monitoring in a Quadrupole Ion-Trap Mass-Spectrometer, Analytical Chemistry, 67 (1995) 3650-3655. [135] F.L. Brancia, B. McCullough, A. Entwistle, J.G. Grossmann, L. Ding, Digital asymmetric waveform isolation (DAWI) in a digital linear ion trap, J Am Soc Mass Spectrom, 21 (2010) 1530-1533.

[136] R. Singh, V. Jayaram, P.T.A. Reilly, Duty cycle-based isolation in linear quadrupole ion traps, Int. J. Mass Spectrom., 343-344 (2013) 45-49.

[137] G.F. Brabeck, P.T.A. Reilly, Mapping ion stability in digitally driven ion traps and guides, International Journal of Mass Spectrometry, 364 (2014) 1-8. [138] G.F. Brabeck, H.J. Chen, N.M. Hoffman, L. Wang, P.T.A. Reilly, Development of MSn in Digitally Operated Linear Ion Guides, Analytical Chemistry, 86 (2014) 77577763.

[139] G.F. Brabeck, H. Koizumi, E. Koizumi, P.T.A. Reilly, Characterization of quadrupole mass filters operated with frequency-asymmetric and amplitudeasymmetric waveforms, International Journal of Mass Spectrometry, 404 (2016) 813.

[140] P.A. Chrisman, S.J. Pitteri, S.A. McLuckey, Parallel ion parking: improving conversion of parents to first-generation products in electron transfer dissociation, Anal Chem, 77 (2005) 3411-3414.

[141] R.G. Cooks, Collision Spectroscopy, Plenum Press, New York and London, 1978.

[142] Q. Dang, F. Xu, L. Wang, X. Huang, X. Dai, X. Fang, R. Wang, C.F. Ding, Theoretical Study of Dual-Direction Dipolar Excitation of Ions in Linear Ion Traps, J Am Soc Mass Spectrom, 27 (2016) 596-606.

[143] J.C. Schwartz, J.E.P. Syka, S.T. Quarmby, in: The 53rd ASMS Conference on Mass Spectrometry and Allied Topics, San Antonio, TX, 2005.

[144] C. Cunningham, Jr., G.L. Glish, D.J. Burinsky, High amplitude short time excitation: a method to form and detect low mass product ions in a quadrupole ion trap mass spectrometer, J Am Soc Mass Spectr, 17 (2006) 81-84.

[145] J. Murrell, D. Despeyroux, S.A. Lammert, J.L. Stephenson, D.E. Goeringer, "Fast excitation" CID in a quadrupole ion trap mass spectrometer, J Am Soc Mass Spectr, 14 (2003) 785-789.

[146] J. Qin, B.T. Chait, Matrix-assisted laser desorption ion trap mass spectrometry: efficient isolation and effective fragmentation of peptide ions, Anal. Chem., 68 (1996) 2108-2112.

[147] O.L. Collin, M. Beier, G.P. Jackson, Dynamic collision-induced dissociation of peptides in a quadrupole ion trap mass spectrometer, Anal. Chem., 79 (2007) 54685473.

[148] U.A. Laskay, O.L. Collin, J.J. Hyland, B. Nichol, G.P. Jackson, S.P. Pasilis, D.C. Duckworth, Dynamic collision-induced dissociation (DCID) in a quadrupole ion trap 
using a two-frequency excitation waveform: II. Effects of frequency spacing and scan rate, J Am Soc Mass Spectr, 18 (2007) 2017-2025.

[149] U.A. Laskay, J.J. Hyland, G.P. Jackson, Dynamic collision-induced dissociation (DCID) in a quadrupole ion trap using a two-frequency excitation waveform: I. Effects of excitation frequency and phase angle, J Am Soc Mass Spectr, 18 (2007) 749-761.

[150] U.A. Laskay, G.P. Jackson, Resonance excitation and dynamic collision-induced dissociation in quadrupole ion traps using higher-order excitation frequencies, Rapid Commun Mass Sp, 22 (2008) 2342-2348.

[151] A.R. Dongre, A. Somogyi, V.H. Wysocki, Surface-induced dissociation: an effective tool to probe structure, energetics and fragmentation mechanisms of protonated peptides, J. Mass Spectrom., 31 (1996) 339-350.

[152] S.A. Lammert, R.G. Cooks, Surface-induced dissociation of molecular ions in a quadrupole ion trap mass spectrometer, J Am Soc Mass Spectr, 2 (1991) 487-491. [153] B.M. Prentice, R.E. Santini, S.A. McLuckey, Adaptation of a 3-D quadrupole ion trap for dipolar DC collisional activation, J Am Soc Mass Spectrom, 22 (2011) 14861492.

[154] B.M. Prentice, S.A. McLuckey, Dipolar DC collisional activation in a "stretched" 3-D ion trap: the effect of higher order fields on rf-heating, J Am Soc Mass Spectr, 23 (2012) 736-744.

[155] L. Wang, F. Xu, C.F. Ding, Dipolar direct current driven collision-induced dissociation in a digital ceramic-based rectilinear ion trap mass spectrometer, Anal Chem, 85 (2013) 1271-1275.

[156] T. Evans-Nguyen, L. Becker, V. Doroshenko, R.J. Cotter, Development of a low power, high mass range mass spectrometer for Mars surface analysis, Int. J. Mass Spectrom., 278 (2008) 170-177.

[157] J.V. Johnson, R.E. Pedder, R.A. Yost, Ms Ms parent scans on a quadrupole ion trap mass-spectrometer by simultaneous resonant excitation of multiple ions, Int. J. Mass Spectrom. Ion Processes, 106 (1991) 197-212.

[158] D.T. Snyder, C.J. Pulliam, R.G. Cooks, Single analyzer precursor scans using an ion trap, Rapid Commun Mass Sp, 30 (2016) 800-804.

[159] M.H. Sonl, R.G. Cooks, Selective injection and isolation of ions in quadrupole ion trap mass spectrometry using notched waveforms created using the inverse fourier transform, Anal. Chem., 66 (1994) 2488-2496.

[160] S. Guan, A.G. Marshall, Stored waveform inverse Fourier transform (SWIFT) ion excitation in trapped-ion mass spectometry - theory and applications, Int. J. Mass Spectrom. Ion Processes, 157/158 (1996) 5-37.

[161] D.T. Snyder, R.G. Cooks, Multigenerational broadband collision-induced dissociation of precursor ions in a linear ion trap, J Am Soc Mass Spectr. [162] D.T. Snyder, P.W. Fedick, R.G. Cooks, Multigenerational collision-induced dissociation for characterization of organic compounds, Anal. Chem., 88 (2016) 9572-9581.

[163] L.L. Lopez, P.R. Tiller, M.W. Senko, J.C. Schwartz, Automated strategies for obtaining standardized collisionally induced dissociation spectra on a benchtop ion trap mass spectrometer, Rapid Commun Mass Sp, 13 (1999) 663-668. 
[164] L. Ding, M. Sudakov, F.L. Brancia, R. Giles, S. Kumashiro, A digital ion trap mass spectrometer coupled with atmospheric pressure ion sources, J. Mass Spectrom., 39 (2004) 471-484.

[165] F.X. Xu, L. Wang, X.H. Dai, X. Fang, C.F. Ding, Resonance Activation and Collision-Induced-Dissociation of Ions Using Rectangular Wave Dipolar Potentials in a Digital Ion Trap Mass Spectrometer, Journal of the American Society for Mass Spectrometry, 25 (2014) 556-562.

[166] F.X. Xu, Q.K. Dang, X.H. Dai, X. Fang, Y.Y. Wang, L. Ding, C.F. Ding, Characteristics of Ion Activation and Collision Induced Dissociation Using Digital Ion Trap Technology, Journal of the American Society for Mass Spectrometry, 27 (2016) 1351-1356.

[167] H.F. Wu, J.S. Brodbelt, Effects of collisional cooling on ion detection in a quadrupole ion trap mass-spectrometer, Int. J. Mass Spectrom. Ion Processes, 115 (1992) 67-81.

[168] D.M. Black, A.H. Payne, G.L. Glish, Determination of cooling rates in a quadrupole ion trap, J Am Soc Mass Spectrom, 17 (2006) 932-938.

[169] A.M. Tabert, M.P. Goodwin, R.G. Cooks, Co-occurrence of boundary and resonance ejection in a multiplexed rectilinear ion trap mass spectrometer, J Am Soc Mass Spectrom, 17 (2006) 56-59.

[170] J.C. Schwartz, J.E. Syka, I. Jardine, High resolution on a quadrupole ion trap mass spectrometer, J Am Soc Mass Spectr, 2 (1991) 198-204.

[171] D.E. Goeringer, W.B. Whitten, J.M. Ramsey, S.A. Mcluckey, G.L. Glish, Theory of high-resolution mass-spectrometry achieved via resonance ejection in the quadrupole ion trap, Anal. Chem., 64 (1992) 1434-1439.

[172] C.G. Yang, M.E. Bier, Investigation of the rapid scan on an electrospray ion trap mass spectrometer, Anal Chem, 77 (2005) 1663-1671.

[173] G. Dobson, J. Murrell, D. Despeyroux, F. Wind, J.C. Tabet, Investigations into the use of a reverse scan in a quadrupole ion trap mass spectrometer, Rapid Commun Mass Sp, 17 (2003) 1657-1664.

[174] V.M. Doroshenko, R.J. Cotter, Linear mass calibration in the quadrupole iontrap mass-spectrometer, Rapid Commun Mass Sp, 8 (1994) 766-776.

[175] L.A. Gill, J.M. Wells, G.E. Patterson, J.W. Amy, R.G. Cooks, Resolution of isobaric and isomeric ions using chemical shifts in an ion trap mass spectrometer, Analytical Chemistry, 70 (1998) 4448-4452.

[176] J.M. Wells, W.R. Plass, G.E. Patterson, O.Y. Zheng, E.R. Badman, R.G. Cooks, Chemical mass shifts in ion trap mass spectrometry: Experiments and simulations, Anal. Chem., 71 (1999) 3405-3415.

[177] E.P. Sheretov, O.W. Rozhkov, D.W. Kiryushin, A.E. Malutin, Mass selective instability mode without a light buffer gas, International Journal of Mass Spectrometry, 191 (1999) 103-111.

[178] W. Xu, Q. Song, S.A. Smith, W.J. Chappell, Z. Ouyang, Ion trap mass analysis at high pressure: a theoretical view, J Am Soc Mass Spectr, 20 (2009) 2144-2153. [179] Q. Song, W. Xu, S.A. Smith, L. Gao, W.J. Chappell, R.G. Cooks, Z. Ouyang, Ion trap mass analysis at high pressure: an experimental characterization, J. Mass Spectrom., 45 (2010) 26-34. 
[180] W.B. Whitten, P.T. Reilly, J.M. Ramsey, High-pressure ion trap mass spectrometry, Rapid Commun Mass Sp, 18 (2004) 1749-1752.

[181] K.H. Blakeman, D. Wolfe, C. Cavanaugh, J.M. Ramsey, High pressure mass spectrometry: The generation of mass spectra at operating pressures exceeding 1 torr in a microscale cylindrical ion trap, Anal. Chem., 88 (2016) 5378-5384.

[182] A.N. Kotana, A.K. Mohanty, A modified auxiliary excitation signal for achieving unidirectional ion ejection in quadrupole ion trap mass spectrometers operating in the resonance ejection mode, International Journal of Mass Spectrometry, 386 (2015) 15-23.

[183] F.A. Londry, J.W. Hager, Mass selective axial ion ejection from a linear quadrupole ion trap, Journal of the American Society for Mass Spectrometry, 14 (2003) 1130-1147.

[184] M. Guna, T.A. Biesenthal, Performance enhancements of mass selective axial ejection from a linear ion trap, J Am Soc Mass Spectrom, 20 (2009) 1132-1140. [185] M. Sugiyama, H. Hasegawa, Y. Hashimoto, Mass-selective axial ejection from a linear ion trap with a direct current extraction field, Rapid Commun Mass Spectrom, 23 (2009) 2917-2922.

[186] A. Moradian, D.J. Douglas, Mass selective axial ion ejection from linear quadrupoles with added octopole fields, J Am Soc Mass Spectrom, 19 (2008) 270280.

[187] R.E. Kaiser, J.N. Louris, J.W. Amy, R.G. Cooks, Extending the mass range of the quadrupole ion trap using axial modulation, Rapid Commun Mass Sp, 3 (1989) 225229.

[188] R.E. Kaiser, R.G. Cooks, G.C. Stafford, J.E.P. Syka, P.H. Hemberger, Operation of a quadrupole ion trap mass-spectrometer to achieve high mass charge ratios, Int. J. Mass Spectrom. Ion Proc., 106 (1991) 79-115.

[189] C.S. Creaser, J.W. Stygall, A comparison of overtone and fundamental resonances for mass range extension by resonance ejection in a quadrupole ion trap mass spectrometer, International Journal of Mass Spectrometry, 191 (1999) 145151.

[190] J. Franzen, R. Gabling, G. Heinen, G. Weiss, Method of mass analyzing a sample by use of a quistor, in, The United States of America as represented by the Secretary of the Army, U.S., 1989.

[191] J. Moxom, P.T. Reilly, W.B. Whitten, J.M. Ramsey, Double resonance ejection in a micro ion trap mass spectrometer, Rapid Commun Mass Sp, 16 (2002) 755-760. [192] J. Franzen, The non-linear ion trap. Part 4. Mass selective instability scan with multipole superposition, Int. J. Mass Spectrom. Ion Processes, 125 (1993) 165-170. [193] V.M. Doroshenko, R.J. Cotter, Effect of phase locking AC and RF voltages for high mass analysis in a quadrupole ion trap mass spectrometer, Rapid Commun Mass Sp, 10 (1996) 1921-1926.

[194] D.T. Snyder, R.G. Cooks, Successive resonances for ion ejection at arbitrary frequencies in an ion trap.

[195] F.A. Londry, R.E. March, Systematic Factors Affecting High Mass-Resolution and Accurate Mass Assignment in a Quadrupole Ion-Trap, International Journal of Mass Spectrometry and Ion Processes, 144 (1995) 87-103. 
[196] E.X. Chen, M. Gehm, R. Danell, M. Wells, J.T. Glass, D. Brady, Compressive mass analysis on quadrupole ion trap systems, J Am Soc Mass Spectr, 25 (2014) 12951304.

[197] X. Zhang, Y. Wang, L. Hu, D. Guo, X. Fang, M. Zhou, W. Xu, Reducing space charge effects in a linear ion trap by rhombic ion excitation and ejection, J Am Soc Mass Spectr, 27 (2016) 1256-1262.

[198] B. Landais, C. Beaugrand, L. Capron-Dukan, M. Sablier, G. Simonneau, C. Rolando, Varying the radio frequency: a new scanning mode for quadrupole analyzers, Rapid Commun Mass Sp, 12 (1998) 302-306.

[199] L. Ding, M. Sudakov, S. Kumashiro, A simulation study of the digital ion trap mass spectrometer, Int. J. Mass Spectrom., 221 (2002) 117-138.

[200] Z. Nie, F. Cui, M. Chu, C.-H. Chen, H.-C. Chang, Y. Cai, Calibration of a frequencyscan quadrupole ion trap mass spectrometer for microparticle mass analysis, Int. J. Mass Spectrom., 270 (2008) 8-15.

[201] Y. Cai, W.P. Peng, S.J. Kuo, H.C. Chang, Calibration of an audio-frequency ion trap mass spectrometer, Int. J. Mass Spectrom., 214 (2002) 63-73.

[202] W.P. Peng, Y. Cai, Y.T. Lee, H.C. Chang, Laser-induced fluorescence/ion trap as a detector for mass spectrometric analysis of nanoparticles, Int. J. Mass Spectrom., 229 (2003) 67-76.

[203] I.C. Lu, J.L. Lin, S.H. Lai, C.H. Chen, Frequency-scanning MALDI linear ion trap mass spectrometer for large biomolecular ion detection, Anal. Chem., 83 (2011) 8273-8277.

[204] U.P. Schlunegger, M. Stoeckli, R.M. Caprioli, Frequency scan for the analysis of high mass ions generated by matrix-assisted laser desorption/ionization in a Paul trap, Rapid Commun Mass Sp, 13 (1999) 1792-1796.

[205] Z. Zhu, C. Xiong, G. Xu, H. Liu, X. Zhou, R. Chen, W.P. Peng, Z. Nie, Characterization of bioparticles using a miniature cylindrical ion trap mass spectrometer operated at rough vacuum, Analyst, 136 (2011) 1305-1309. [206] L. Ding, S. Kumashiro, Ion motion in the rectangular wave quadrupole field and digital operation mode of a quadrupole ion trap mass spectrometer, Rapid Commun Mass Sp, 20 (2006) 3-8.

[207] A. Berton, P. Traldi, L. Ding, F.L. Brancia, Mapping the stability diagram of a digital ion trap (DIT) mass spectrometer varying the duty cycle of the trapping rectangular waveform, J Am Soc Mass Spectrom, 19 (2008) 620-625. [208] D. Wang, F.H. van Amerom, T. Evans-Nguyen, High-speed digital frequency scanning ion trap mass spectrometry, Anal. Chem., 85 (2013) 10935-10940. [209] B. Xue, L. Ma, L. Sun, W. Gao, P. Cheng, L. Ding, Z. Huang, Z. Zhou, Resonance ejection mass scan using dipole excitation with a non-integer frequency ratio in a digital linear ion trap mass spectrometer, Rapid Commun Mass Sp, 30 (2016) 1985 1990.

[210] E.R. Badman, R.G. Cooks, Cylindrical ion trap array with mass selection by variation in trap dimensions, Anal. Chem., 72 (2000) 5079-5086.

[211] B.M. Prentice, S.A. McLuckey, Analysis of high mass-to-charge ions in a quadrupole ion trap mass spectrometer via an end-cap quadrupolar direct current downscan, Anal Chem, 84 (2012) 7562-7569. 
[212] J.F.J. Todd, A.D. Penman, R.D. Smith, Some Alternative Scanning Methods for the Ion Trap Mass-Spectrometer, International Journal of Mass Spectrometry and Ion Processes, 106 (1991) 117-135.

[213] D.T. Snyder, C.J. Pulliam, R.G. Cooks, Calibration procedure for secular frequency scanning in an ion trap, Rapid Commun Mass Sp, 30 (2016) 1190-1196. [214] D.T. Snyder, C.J. Pulliam, R.G. Cooks, Linear mass scans in quadrupole ion traps using the inverse Mathieu q scan, Rapid Commun Mass Sp, 30 (2016) 2369-2378. [215] D.T. Snyder, C.J. Pulliam, R.G. Cooks, Extending the mass range of a miniature mass spectrometer using the inverse Mathieu q scan.

[216] C. Zhang, H. Chen, A.J. Guymon, G. Wu, R.G. Cooks, Z. Ouyang, Instrumentation and methods for ion and reaction monitoring using a non-scanning rectilinear ion trap, International Journal of Mass Spectrometry, 255-256 (2006) 1-10. [217] B. Brkić, S. Giannoukos, N. France, R. Murcott, F. Siviero, S. Taylor, Optimized DLP linear ion trap for a portable non-scanning mass spectrometer, International Journal of Mass Spectrometry, 369 (2014) 30-35.

[218] A. Janulyte, Y. Zerega, J. Andre, B. Brkic, S. Taylor, Performance Assessment of a Portable Mass Spectrometer Using a Linear Ion Trap Operated in Non-Scanning Mode, Rapid Commun Mass Spectrom, (2016).

[219] W.H. Benner, A gated electrostatic ion trap to repetitiously measure the charge and m/z of large electrospray ions, Analytical Chemistry, 69 (1997) 41624168.

[220] D.Z. Keifer, D.L. Shinholt, M.F. Jarrold, Charge Detection Mass Spectrometry with Almost Perfect Charge Accuracy, Anal Chem, 87 (2015) 10330-10337. [221] W.P. Peng, H.C. Lin, H.H. Lin, M. Chu, A.L. Yu, H.C. Chang, C.H. Chen, Chargemonitoring laser-induced acoustic desorption mass spectrometry for cell and microparticle mass distribution measurement, Angew Chem Int Edit, 46 (2007) 3865-3869.

[222] W.P. Peng, H.C. Lin, M.L. Chu, H.C. Chang, N.H. Lin, A.L. Yu, C.H. Chen, Charge monitoring cell mass spectrometry, Anal. Chem., 80 (2008) 2524-2530.

[223] W.-P. Peng, S.-W. Chou, A.A. Patil, Measuring masses of large biomolecules and bioparticles using mass spectrometric techniques, Analyst, 139 (2014) 3507-3523.

[224] M. Soni, V. Frankevich, M. Nappi, R.E. Santini, J.W. Amy, R.G. Cooks, BroadBand Fourier Transform Quadrupole Ion Trap Mass Spectrometry, Anal. Chem., 68 (1996) 3314-3320.

[225] A.G. Marshall, C.L. Hendrickson, G.S. Jackson, Fourier transform ion cyclotron resonance mass spectrometry: A primer, Mass Spectrometry Reviews, 17 (1998) 135.

[226] R.H. Perry, R.G. Cooks, R.J. Noll, Orbitrap mass spectrometry: Instrumentation, ion motion and applications, Mass Spectrometry Reviews, 27 (2008) 661-699. [227] M. Soni, V. Frankevich, M. Nappi, R.E. Santini, J.W. Amy, R.G. Cooks, BroadBand Fourier Transform Quadrupole Ion Trap Mass Spectrometry, Anal. Chem., 68 (1996) 3314-3320.

[228] S.D. Fuerstenau, W.H. Benner, J.J. Thomas, C. Brugidou, B. Bothner, G. Siuzdak, Mass spectrometry of an intact virus, Angew Chem Int Edit, 40 (2001) 542-544. 
[229] Z. Nie, F. Cui, Y.K. Tzeng, H.C. Chang, M. Chu, H.C. Lin, C.H. Chen, H.H. Lin, A.L. $\mathrm{Yu}$, High-speed mass analysis of whole erythrocytes by charge-detection quadrupole ion trap mass spectrometry, Anal. Chem., 79 (2007) 7401-7407.

[230] Z. Nie, F. Cui, M. Chu, C.H. Chen, H.C. Chang, Y. Cai, Calibration of a frequencyscan quadrupole ion trap mass spectrometer for microparticle mass analysis, International Journal of Mass Spectrometry, 270 (2008) 8-15. [231] S.-W. Chou, G.-R. Shiu, H.-C. Chang, W.-P. Peng, Wavelet-Based Method for Time-Domain Noise Analysis and Reduction in a Frequency-Scan Ion Trap Mass Spectrometer, Journal of The American Society for Mass Spectrometry, 23 (2012) 1855-1864.

[232] S. Guan, A.G. Marshall, Stored waveform inverse Fourier transform axial excitation/ejection for quadrupole ion trap mass spectrometry, Anal. Chem., 65 (1993) 1288-1294.

[233] S. Guan, A.G. Marshall, Stored waveform inverse Fourier transform (SWIFT) ion excitation in trapped-ion mass spectometry: Theory and applications, International Journal of Mass Spectrometry and Ion Processes, 157 (1996) 5-37. [234] M. Nappi, V. Frankevich, M. Soni, R.G. Cooks, Characteristics of a broad-band Fourier transform ion trap mass spectrometer, International Journal of Mass Spectrometry, 177 (1998) 91-104.

[235] N.L. Kelleher, M.W. Senko, M.M. Siegel, F.W. McLafferty, Unit resolution mass spectra of $112 \mathrm{kDa}$ molecules with 3 Da accuracy, Journal of the American Society for Mass Spectrometry, 8 (1997) 380-383.

[236] A. Makarov, Electrostatic Axially Harmonic Orbital Trapping: A HighPerformance Technique of Mass Analysis, Anal. Chem., 72 (2000) 1156-1162. [237] M. Hardman, A.A. Makarov, Interfacing the Orbitrap Mass Analyzer to an Electrospray Ion Source, Anal. Chem., 75 (2003) 1699-1705.

[238] A. Makarov, E. Denisov, A. Kholomeev, W. Balschun, O. Lange, K. Strupat, S. Horning, Performance Evaluation of a Hybrid Linear Ion Trap/Orbitrap Mass Spectrometer, Anal. Chem., 78 (2006) 2113-2120.

[239] C. Köster, Twin Trap or Hyphenation of a 3D Paul- and a Cassinian Ion Trap, Journal of The American Society for Mass Spectrometry, 26 (2015) 390-396.

[240] C. Köster, The concept of electrostatic non-orbital harmonic ion trapping, International Journal of Mass Spectrometry, 287 (2009) 114-118.

[241] Q. Hu, A.A. Makarov, R.G. Cooks, R.J. Noll, Resonant ac Dipolar Excitation for Ion Motion Control in the Orbitrap Mass Analyzer, The Journal of Physical Chemistry A, 110 (2006) 2682-2689.

[242] Q. Hu, R.G. Cooks, R.J. Noll, Phase-enhanced selective ion ejection in an orbitrap mass spectrometer, Journal of the American Society for Mass Spectrometry, 18 (2007) 980-983.

[243] R.H. Perry, Q. Hu, G.A. Salazar, R.G. Cooks, R.J. Noll, Rephasing ion packets in the orbitrap mass analyzer to improve resolution and peak shape, Journal of the American Society for Mass Spectrometry, 20 (2009) 1397-1404.

[244] M.E. Belov, E. Damoc, E. Denisov, P.D. Compton, S. Horning, A.A. Makarov, N.L. Kelleher, From Protein Complexes to Subunit Backbone Fragments: A Multi-stage Approach to Native Mass Spectrometry, Anal. Chem., 85 (2013) 11163-11173. 
[245] S. Rosati, R.J. Rose, N.J. Thompson, E. van Duijn, E. Damoc, E. Denisov, A. Makarov, A.J.R. Heck, Exploring an Orbitrap Analyzer for the Characterization of Intact Antibodies by Native Mass Spectrometry, Angewandte Chemie International Edition, 51 (2012) 12992-12996.

[246] J.J. Barroso, M.O. Terra, Beam transport in a quadrupole electrostatic system, Brazilian Journal of Physics, 34 (2004) 1598-1601.

[247] Y. Wang, J. Franzen, K.P. Wanczek, The non-linear resonance ion trap. Part 2. A general theoretical analysis, Int. J. Mass Spectrom. Ion Processes, 124 (1993) 125144.

[248] D.T. Snyder, C.J. Pulliam, Z. Ouyang, R.G. Cooks, Miniature and fieldable mass spectrometers: Recent advances, Anal. Chem., 88 (2016) 2-29.

[249] R.K. Julian, R.G. Cooks, Broad-band excitation in the quadrupole ion-trap mass-spectrometer using shaped pulses created with the inverse fourier-transform, Anal. Chem., 65 (1993) 1827-1833.

\section{Figures}



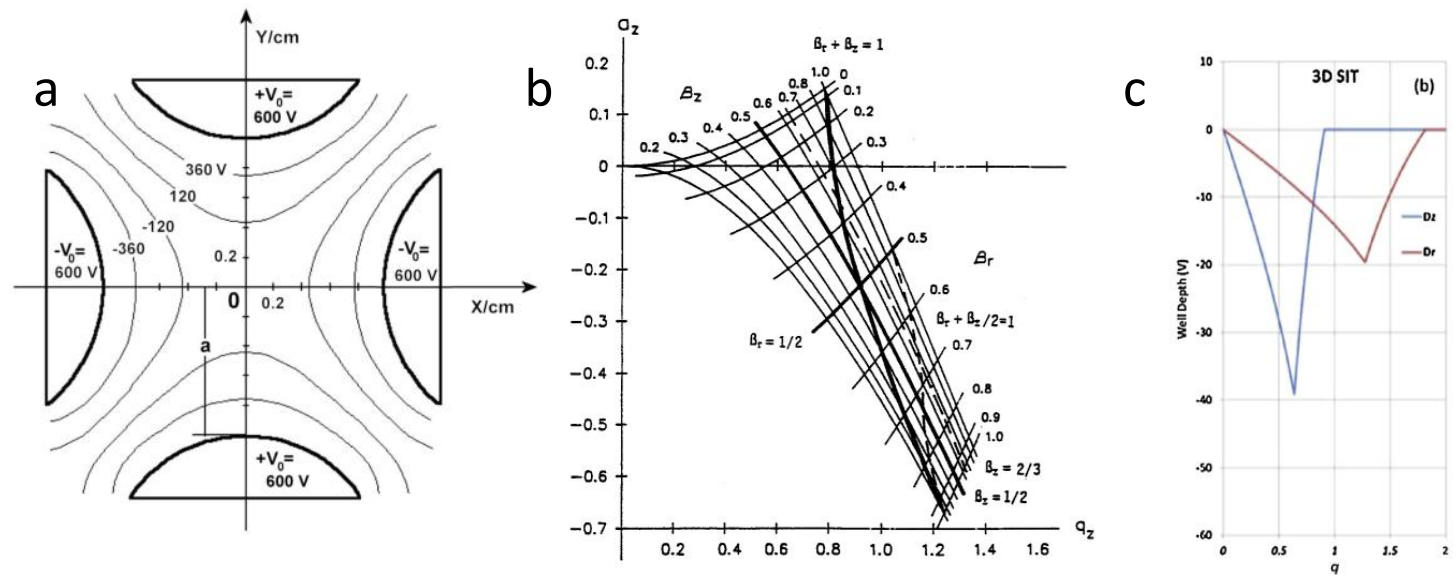

Figure 1: (a) Cross-section of quadrupole rods and equipotential contours created by a quadrupole potential [246], (b) Mathieu stability diagram with iso- $\beta$ (i.e. $\beta_{\mathrm{r}}$ and $\beta_{z}$ ) and nonlinear resonance lines [247], and (c) pseudopotential well depth in $r$ and $\mathrm{z}$ directions in a 3D quadrupole ion trap (SIT = sinusoidal ion trap) [47]. 


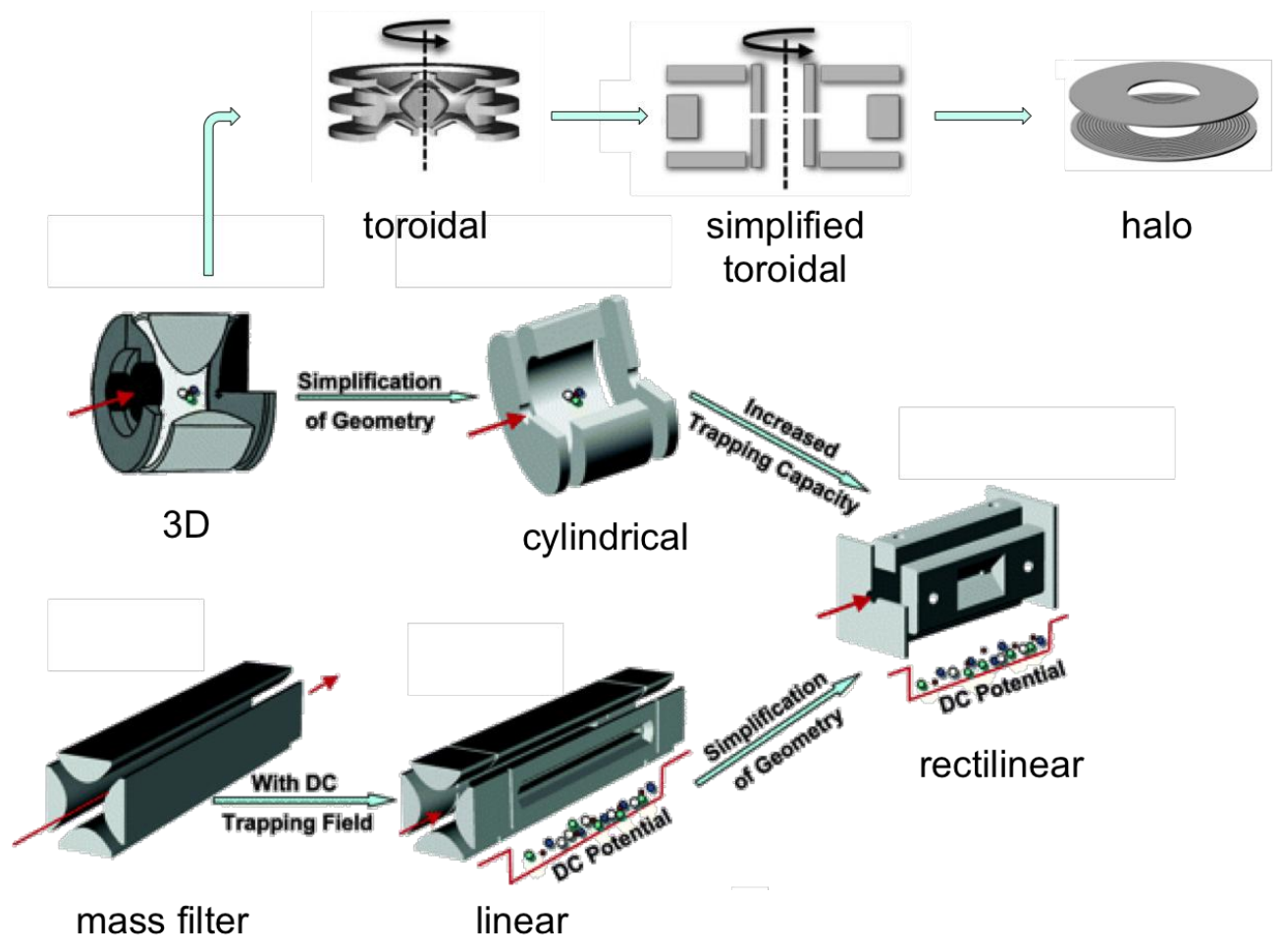

Figure 2: Evolution of ion trap mass analyzers. Adapted from ref. [248]. 

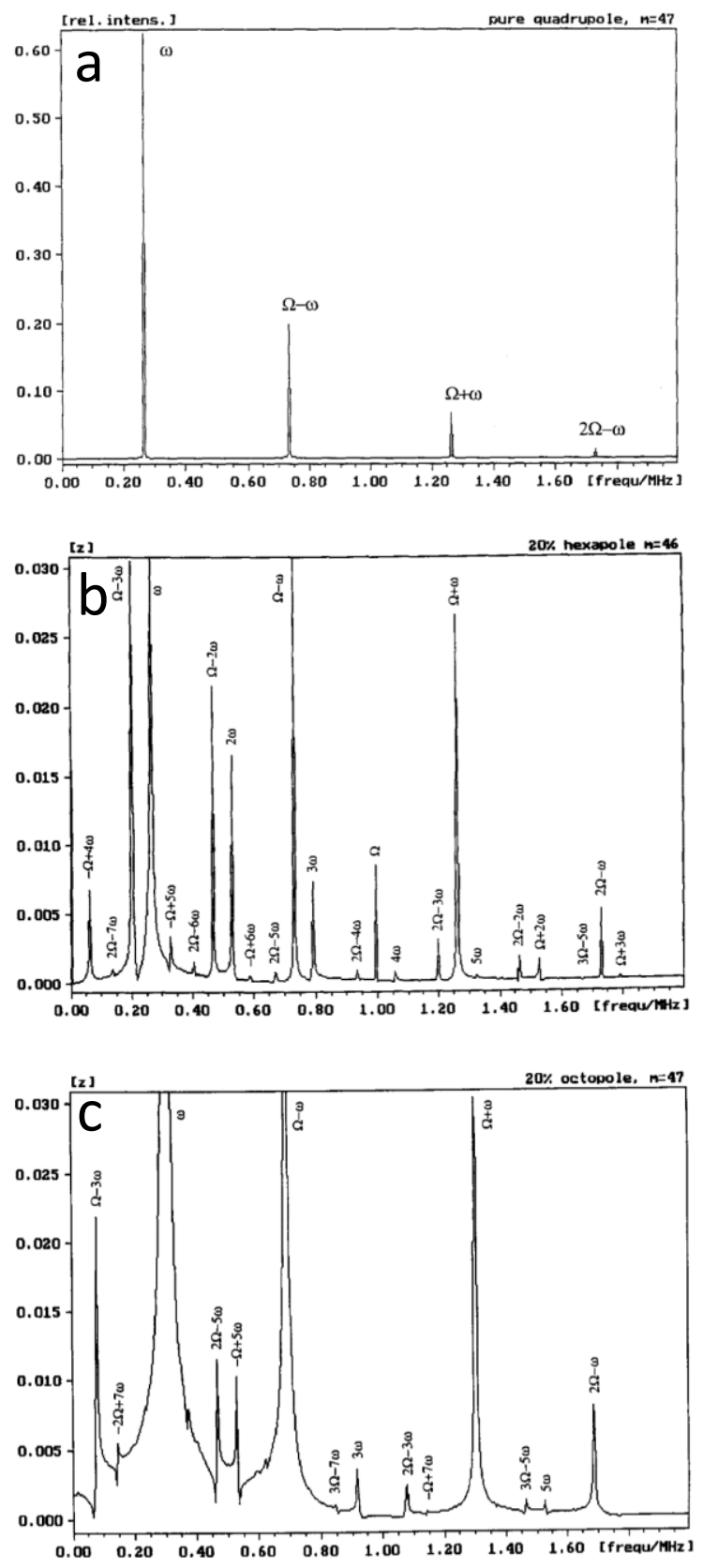

Figure 3: Frequency domain analysis of ions in (a) pure quadrupole field, (b) quadrupole field with $20 \%$ hexapole field superposition, and (c) quadrupole field with 20\% octopole field superposition [66]. 

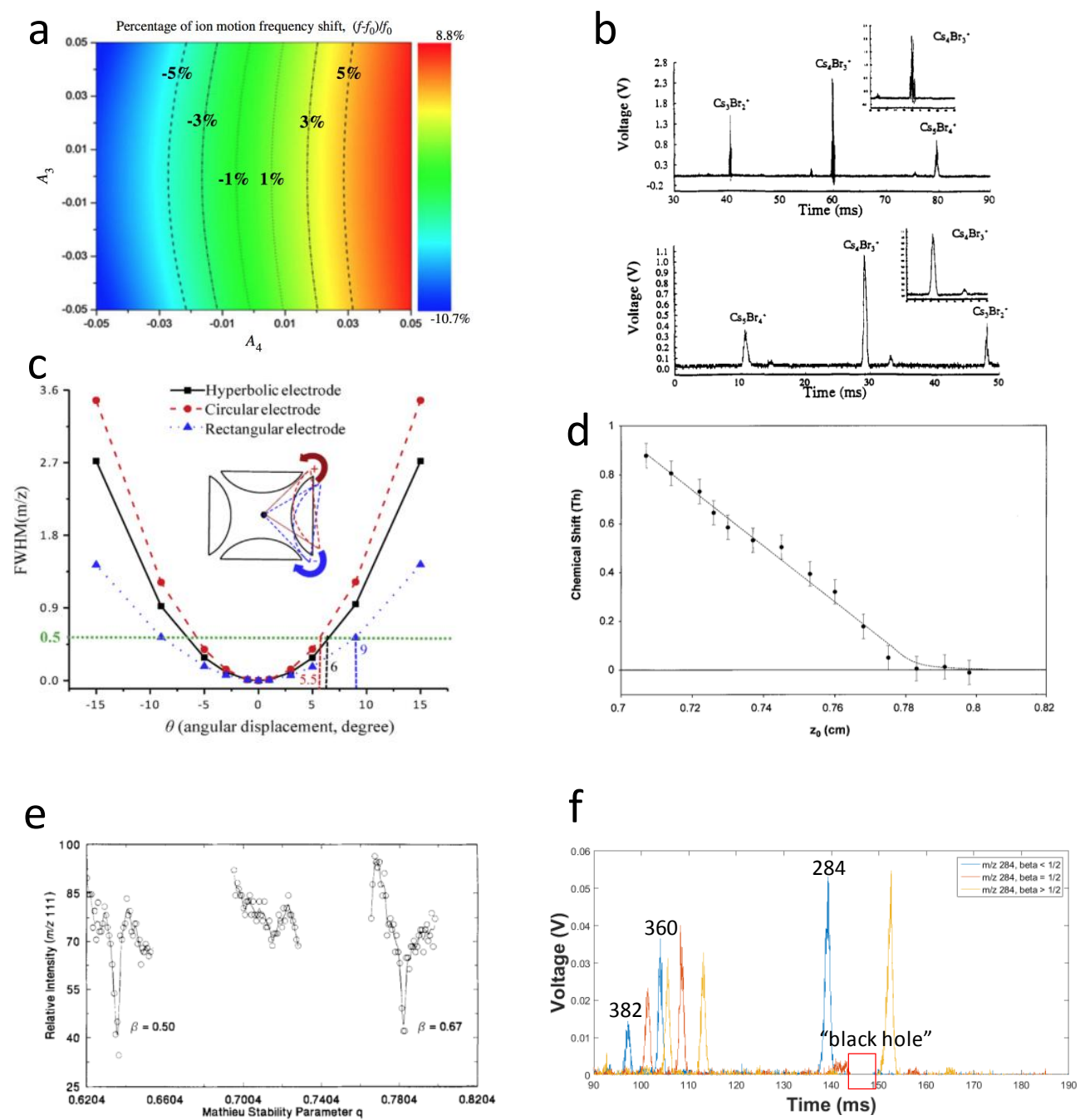

Figure 4: Effect of higher order fields on ion trap operation: (a) ion motion frequency shift with added hexapole and octopole fields [77], (b) effect of frequency shift on sensitivity and resolution in forward (top) and reverse (bottom) resonance ejection [87], (c) resolution as a function of electrode angular displacement (inducing nonlinear fields) [90], (d) chemical mass shift as a function of trap radius (i.e. higher-order field contribution) [176], (e) intensity of product ion $m / z 111$ produced from collision-induced dissociation of 1,2-dichlorobenzene as a function of the product ion's qu value, indicating loss of product ions at nonlinear resonance 
points [111], and (f) blank intensity profile for $m / z 284$ when placed on a nonlinear resonance line, observed using a secular frequency scan [114]. 

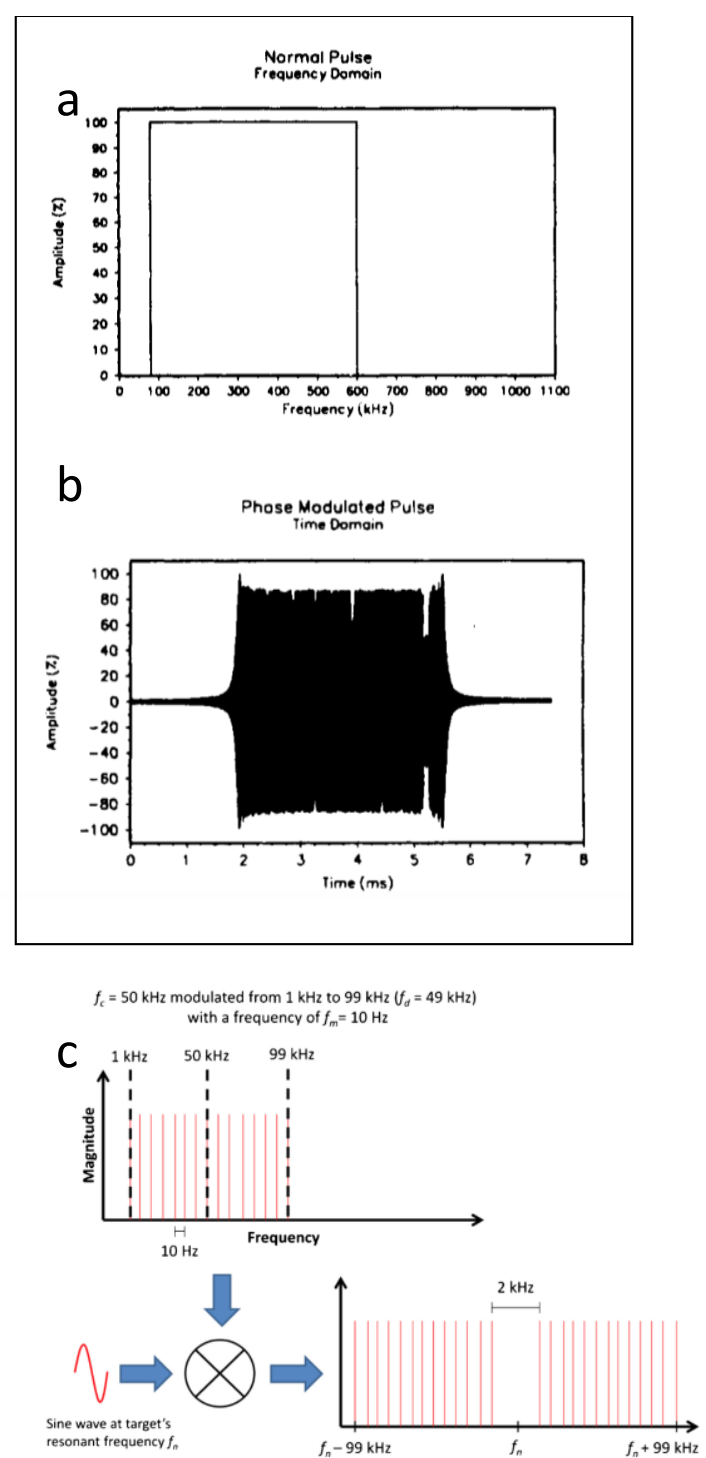

d
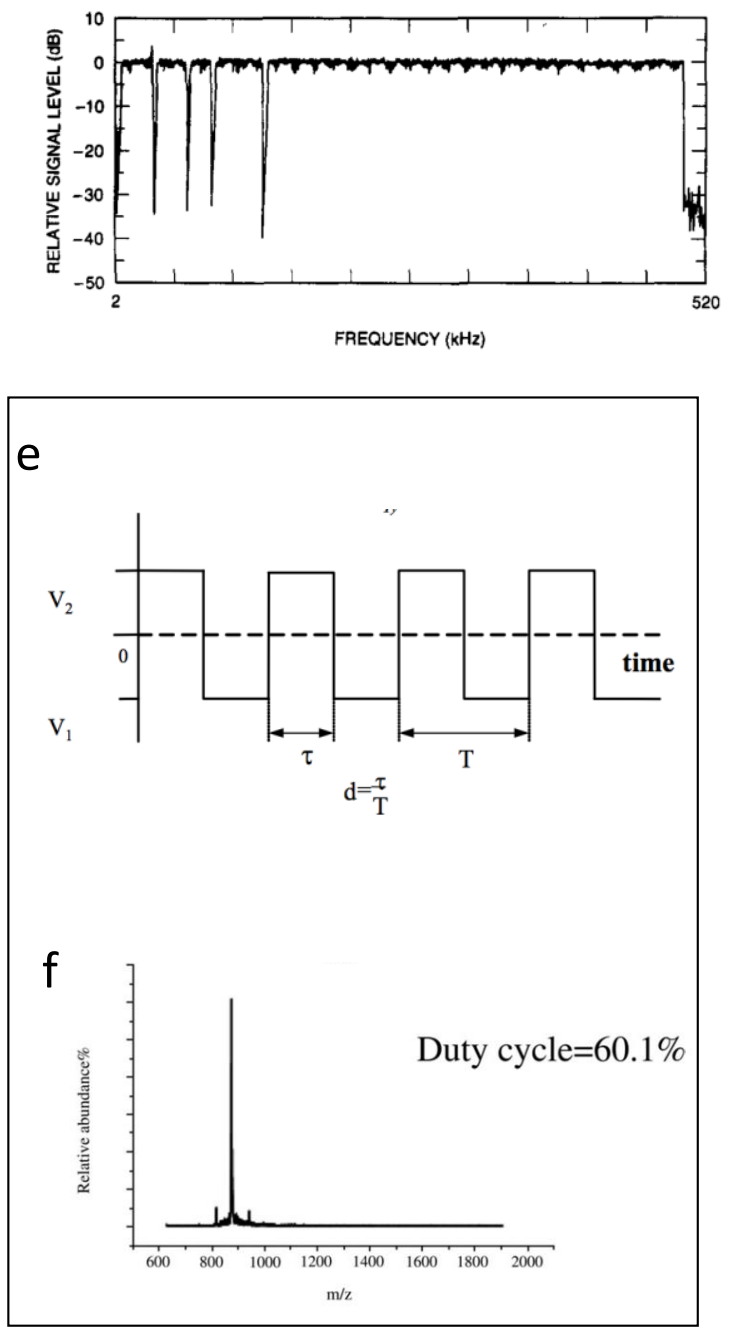

Figure 5: Methods of isolation in a quadrupole ion trap: (a) and (b) synthesis of SWIFT waveform and conversion from frequency domain to time domain [249], (c) broadband excitation with frequency modulation and mixing [130], (d) filtered noise field frequency spectrum [132], (e) and (f) isolation in a digital ion trap using digital asymmetric waveforms [135]. 


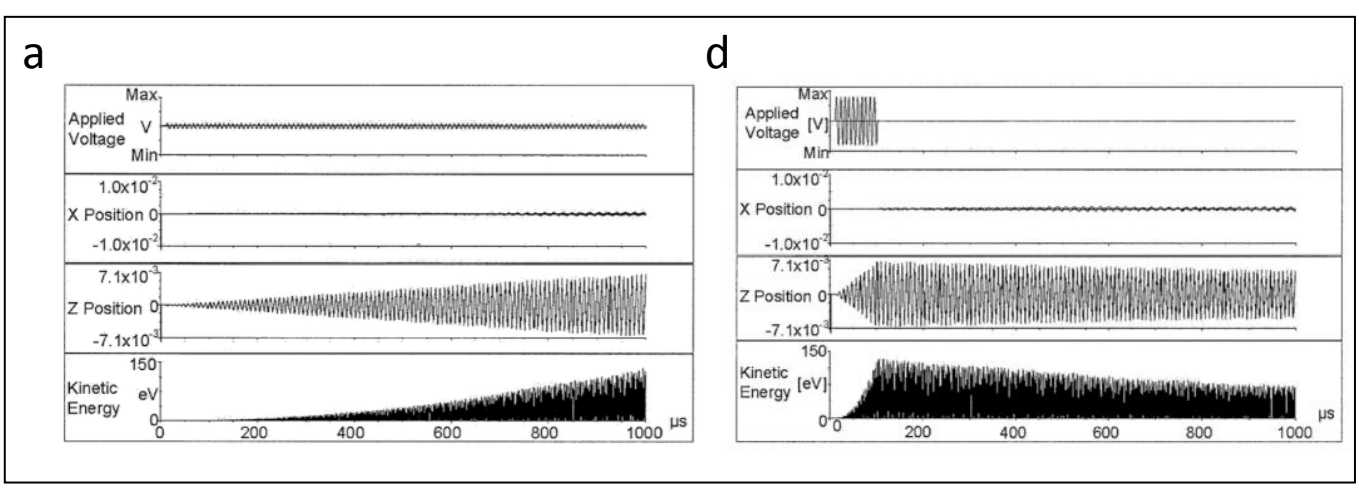

\section{b}
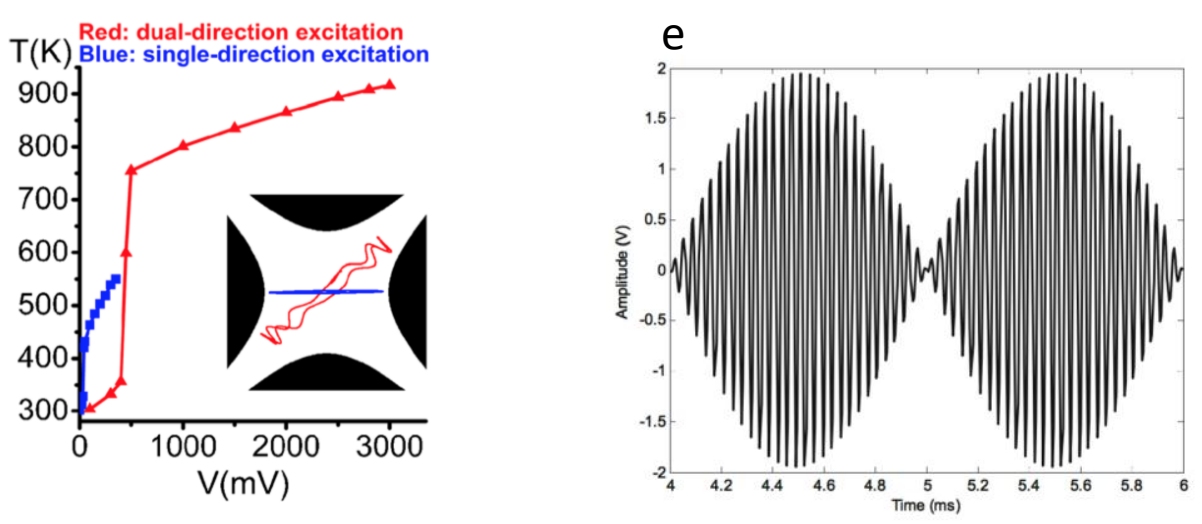

C

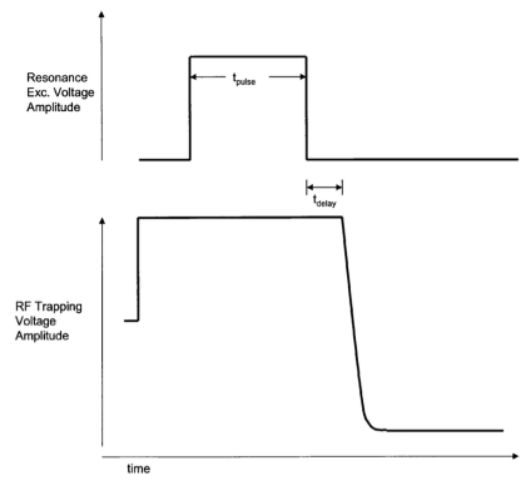

Figure 6: Methods of activation in a quadrupole ion trap: (a) conventional resonance excitation compared to (d) "fast excitation" CID [145], (b) simulation of ion temperature with rhombic (dual direction) ion excitation (red) compared to single direction excitation (blue) [142], (c) pulsed Q dissociation [100, 143], (e) dynamic collision-induced dissociation excitation waveform from superposition of 
171 and $172 \mathrm{kHz}$ [149], and (f) multigenerational collision-induced dissociation

(Wideband Activation) [162].

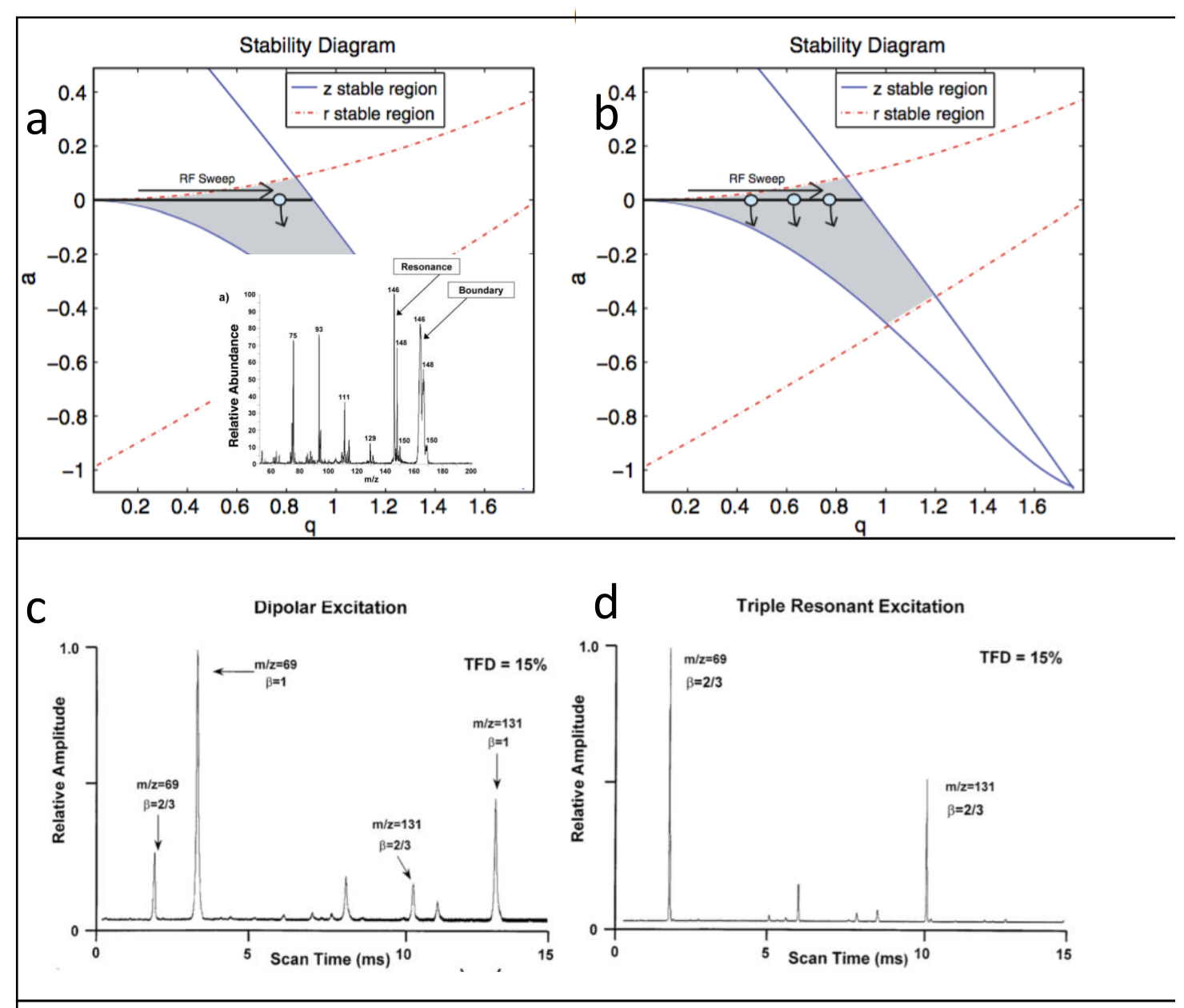


Figure 7: Methods of ion ejection in quadrupole ion traps: (a) resonance ejection and (b) compressive mass spectrometry [196], (c) double and (d) triple resonance ejection [84], (e) mass spectrum from resonance ejection with inset ion motion [197], and (f) mass spectrum from rhombic ejection with inset ion motion [197]. 


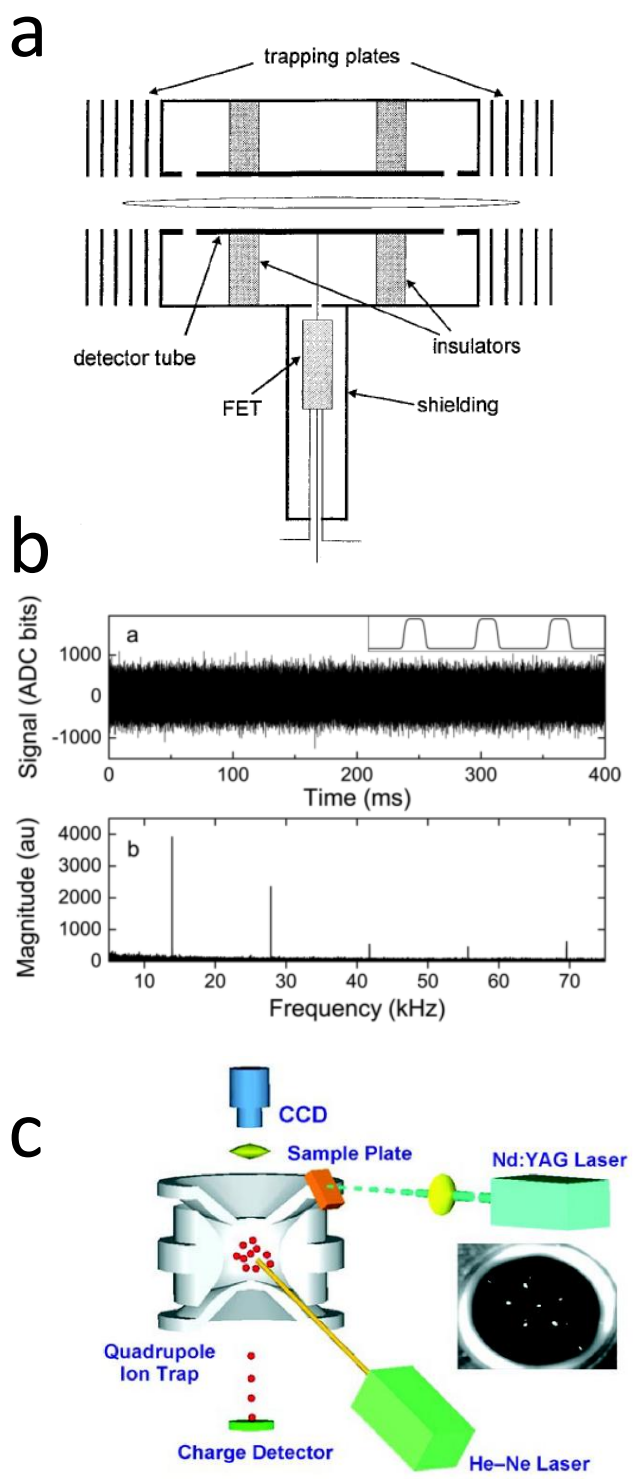

Figure 8: Image charge detection (induced voltages): a) schematic diagram of the gated electrostatic ion trap [219]; b) charge detection mass spectrometry, inset a) time domain signal, inset b) Fourier transform of time domain, giving ion frequencies of motion and hence m/z [220]; c) experimental setup of charge detection quadrupole ion trap mass spectrometer with laser-induced acoustic desorption source [221]. 

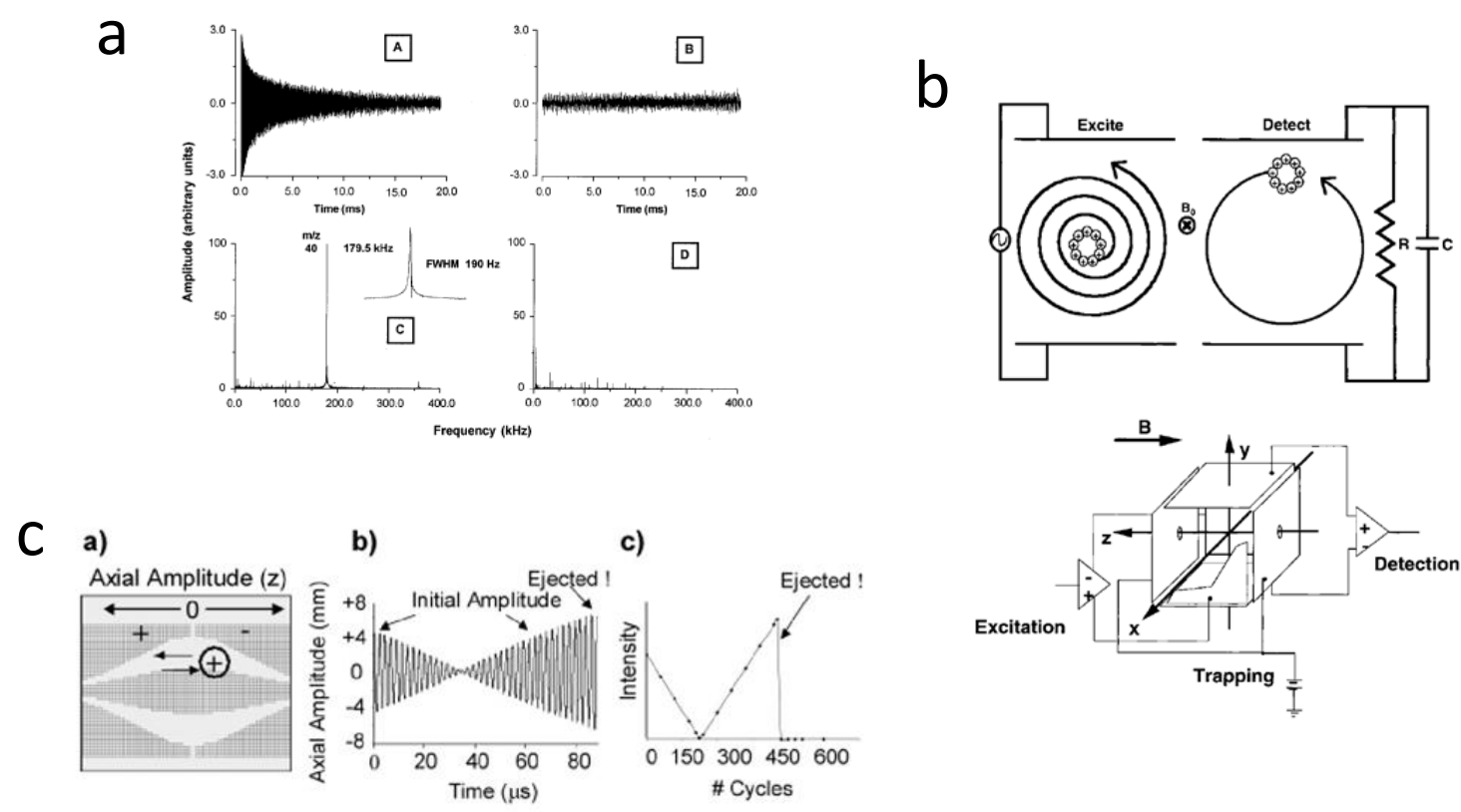

Figure 9: Image charge detection (induced currents): a) the time domain signal and background transients (A, B) and their FFTs (C, D) in a quadrupole ion trap [224]; b) incoherent ion cyclotron orbital motion (top left) is converted to coherent motion (top right) by the application of a rotating electric field and at the ICR frequency of the ions of a given $\mathrm{m} / \mathrm{z}$ value. The electronic circuitry is shown in the bottom diagram [225]; c) a resonant ac waveform applied $180^{\circ}$ with respect to ion oscillation, inset (a) schematic depiction of outer electrode polarity required to achieve $180^{\circ}$ phase relative to a positive ion by SIMION. The outer electrode is split at the equator, the central plane in the Orbitrap $(z=0)$, inset (b) SIMION simulation results for axial position vs. time for a single ion undergoing resonant ac excitation. Inset (c) ITSIM simulation results of peak intensity as a function of number of cycles of applied ac for a single ion system [241]. 


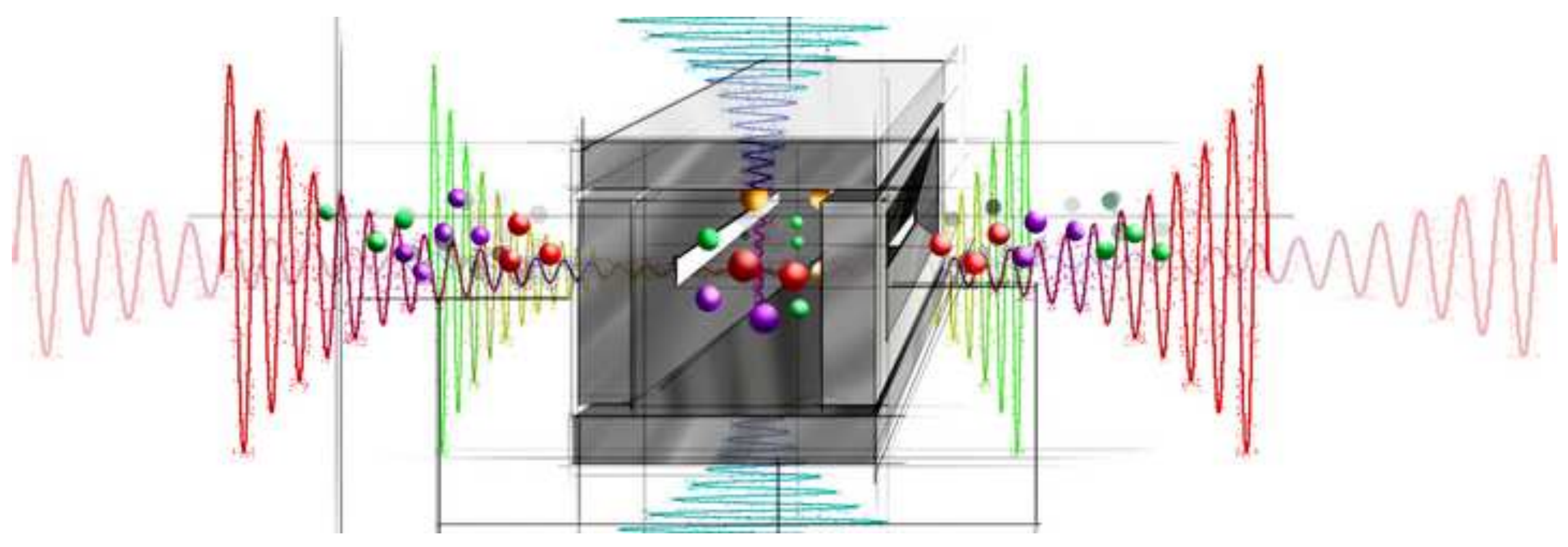

